\title{
I-mode studies at ASDEX Upgrade: L-I and I-H transitions, pedestal and confinement properties
}

\author{
F. Ryter ${ }^{1}$, R. Fischer ${ }^{1}$, J.C. Fuchs ${ }^{1}$, T. Happel ${ }^{1}$, R.M. McDermott ${ }^{1}$, E. \\ Viezzer $^{1}+$, E. Wolfrum ${ }^{1}$, L. Barrera Orte ${ }^{1} \S$, M. Bernert ${ }^{1}$, A. Burckhart ${ }^{1}$, \\ S. da Graça ${ }^{2} \|$, B. Kurzan ${ }^{1}$, P. McCarthy ${ }^{3}$, T. Pütterich ${ }^{1}$, W. Suttrop ${ }^{1}$, M. \\ Willensdorfer $^{1}$ and the ASDEX Upgrade Team ${ }^{1}$ \\ ${ }^{1}$ Max-Planck-Institut für Plasmaphysik, Boltzmannstr. 2, 85748 Garching, Germany \\ 2 Instituto de Plasmas e Fusão Nuclear, Instituto Superior Técnico/UTL, Lisboa, Portugal \\ ${ }^{3}$ Department of Physics, University College Cork, Cork, Ireland \\ E-mail: ryter@ipp.mpg · de
}

\begin{abstract}
The I-mode is a plasma regime obtained when the usual L-H power threshold is high, e.g. with unfavourable ion $\nabla B$ direction. It is characterised by the development of a temperature pedestal while the density remains roughly as in the L-mode. This leads to a confinement improvement above the L-mode level which can sometimes reach H-mode values. This regime, already obtained in the ASDEX Upgrade tokamak about two decades ago, has been studied again since 2009 taking advantage of the development of new diagnostics and heating possibilities. The I-mode in ASDEX Upgrade has been achieved with different heating methods such as NBI, ECRH and ICRF. The I-mode properties, power threshold, pedestal characteristics and confinement, are independent of the heating method. The power required at the L-I transition exhibits an offset linear density dependence but, in contrast to the L-H threshold, depends weakly on the magnetic field. The L-I transition seems to be mainly determined by the edge pressure gradient and the comparison between ECRH and NBI induced L-I transitions suggests that the ion channel plays a key role. The I-mode often evolves gradually over a few confinement times until the transition to H-mode which offers a very interesting situation to study the transport reduction and its link with the pedestal formation. Exploratory discharges in which $n=2$ magnetic perturbations have been applied indicate that these can lead to an increase of the I-mode power threshold by flattening the edge pressure at fixed heating input power: more heating power is necessary to restore the required edge pressure gradient. Finally, the confinement properties of the I-mode are discussed in detail.
\end{abstract}

‡ Present affiliation: Dept. of Atomic, Molecular and Nuclear Physics, University of Seville, Seville, Spain

$\S$ Present affiliation: ITER Physics Department, EUROfusion Programme - Physics Management Unit

|| Present address: Diamond Light Source, Harwell Science and Innovation Campus Didcot OX11 0DE England 


\section{Introduction}

The H-mode threshold power, $P_{\text {thres }}$, is well-known to be low when the ion $\nabla B$ drift is towards the X-point, while it is about two times higher when this drift is directed away from the X-point. The latter case is often named unfavourable configuration in contrast to the former considered as favourable because good confinement is easily achieved at low power. In addition, as also well-known, $P_{\text {thres }}$ is about two times higher in hydrogen compared to deuterium in both configurations. These high $P_{\text {thres }}$ cases provide conditions to study Lmodes over a wider range of heating power than in the usual favourable cases. Earlier investigations at ASDEX Upgrade, [1, 2], revealed that in these scenarios, including hydrogen in the favourable direction, the power degradation of the confinement time was much weaker than $\tau_{E} \propto P^{-0.7}$ predicted by confinement scalings used at that time for the L-mode and $\mathrm{H}$ mode, [3] and [4] respectively. Such plasmas exhibit improved confinement at high heating power, but did not feature the classical transition to H-mode and were named "Improved Lmodes". The weak power degradation was found to be caused by an increase of the edge temperatures with a concomitant steepening of the edge gradients, building a temperature pedestal similar to that of the H-mode, but the density profile almost did not change, the edge density profile remained very similar to that of the L-mode, [5]. Thus, these plasmas exhibit an edge transport barrier for heat but not for particle transport. The transition to H-mode in which particle transport is also reduced was achieved at powers which are about two times above the usual power threshold. The studies on the Improved L-mode at ASDEX Upgrade have not been continued beyond 1996, mainly due to technical restrictions on the possibilities to operate with the unfavourable ion $\nabla B$ drift.

During the last years, the Improved L-mode has been extensively studied in Alcator C-Mod and this mode is now labelled "I-mode", [6, 7, 8, 9]. Therefore, we also use the label I-mode throughout this paper. It should be underlined that I-mode should not be confused with Iphase which is a plasma behaviour observed prior to the L-H transition, under the favourable threshold conditions, characterised by limit cycle oscillations attributed to the modulation of the turbulence level through a predator-prey mechanism between turbulence and self-induced zonal flows (ZFs) and/or geodesic acoustic modes (GAMs), see e.g. [10, 11] for ASDEX Upgrade.

In Alcator C-Mod, the transition from L to I mode is generally accompanied by the appearance of the weakly coherent mode (WCM) at about $300 \mathrm{kHz}$, located radially at the very edge of 
the plasma, in the steep gradient region of the temperature pedestal, $[7,8,12]$. The WCM is seen in the density fluctuations measured by reflectometry and in the electron temperature fluctuations. More recently it has been shown that, in parallel to the WCM, a GAM also develops at about $10 \mathrm{kHz}$ and that WCM, GAM and turbulence are non-linearly coupled, [13]. This phenomenology is also observed in ASDEX Upgrade, [14]. Due to the formation of the temperature pedestal the radial electric field well $\left(E_{r}\right)$ at the plasma edge deepens, its minimum becomes more negative. The level of the turbulence fluctuations decreases during the development of the I-mode which probably explains the formation of the pedestal. But it is not clarified yet whether the transition to I-mode and the correlated heat transport reduction is caused by the WCM-GAM impact on turbulence, or by the radial electric field, or by a combination of all these effects. At Alcator C-Mod, the observed lack of transport reduction in the particle channel is attributed to the presence of the WCM.

Stimulated by the I-mode studies performed at Alcator C-Mod we restarted I-mode investigations at ASDEX Upgrade in 2009. This was justified by the extension of the experimental and diagnostic possibilities since our last Improved L-mode studies which date back to 1996. The new experimental capabilities include in particular the availability of enough electron heating power through the electron cyclotron resonance heating (ECRH) system to reach the I-mode and therefore to investigate pure electron heating versus mixed ionelectron provided by neutral beam injection (NBI). On the diagnostic side, the installation and upgrade of the core and edge active Charge Exchange Recombination Spectroscopy (CXRS) diagnostics provide detailed $T_{i}$ and rotation profiles. Further, Doppler reflectometry, also named back-scattering reflectometry, has been installed, which provides measurements of turbulence and perpendicular propagation velocity of density fluctuations, as discussed in more detail below.

The I-mode combines the advantage of good confinement without the drawback of the ELMs whose power load in the divertor is a serious issue for future tokamak reactors. However, it is reached at a heating power which is generally above the H-mode threshold in the favourable configuration also foreseen for ITER, and might therefore not be accessible in ITER, as the available heating power might not be sufficient. In addition, as will be shown below, the I-mode often evolves slowly over several confinement times, even at constant input power and therefore in an uncontrolled manner, until a transition to H-mode occurs. This lack of control on the plasma regime might be quite problematic for a fusion reactor. However, even 
if the I-mode may not be considered presently as a promising scenario for ITER, it should be emphasised that the development of the I-mode temperature pedestal, together with the decoupling of heat and particle transport channels, as well as the transition from this state to $\mathrm{H}$-mode, extend the possibilities of investigating pedestal physics and transport reduction to conditions which are different from the usual H-mode and might yield a deeper understanding of these mechanisms.

This is the main motivation of the present work in which we describe and discuss the experimental results obtained on the I-mode in ASDEX Upgrade since 2009. The paper is structured as follows. In the next section the experimental conditions and the most important diagnostics used for the present work are presented. In section 3 we describe the main experimental characteristics of the I-mode discharges under different conditions, in particular comparison between ECRH and NBI. In section 4 we discuss the properties of the I-mode pedestal. Section 5 is dedicated to the features of the L-I and I-H transitions, while the confinement properties are discussed in section 6 . The paper is completed by the summary and conclusion.

\section{Experimental conditions and diagnostics}

ASDEX Upgrade is a divertor tokamak of nominal major radius $R=1.65 \mathrm{~m}$ and minor radius $a=0.5 \mathrm{~m}$, while the usual plasmas have an elongation of about 1.6. The device is equipped with NBI, ECRH, as well as heating possibilities in the ion cyclotron resonance frequency range (ICRF) whereby the hydrogen minority scheme is the standard heating method. The experiments presented here were carried out in deuterium.

The NBI at $60 \mathrm{kV}$ has been used, up to two beams with heating power of $2.5 \mathrm{MW}$ each. The ECRH was deposited in the central part of the plasma, $\rho_{\text {pol }}<0.25$, where $\rho_{\text {pol }}$ is the square root of the normalised poloidal flux. This normalised flux radius will be used throughout the paper. The ECRH scheme was X2 which provides $100 \%$ absorption in the electron channel with a narrow deposition profile [15]. The system provides up to $3.2 \mathrm{MW}$ since the 2011 campaign, allowing to explore the L-I transition and the I-mode with pure electron heating up to the transition into H-mode.

As mentioned above, the I-mode usually appears when enough power is applied in configurations with high $\mathrm{H}$-mode power threshold, in most of the cases with the ion $\nabla B$ drift directed away from the X-point. At ASDEX Upgrade, the unfavourable ion $\nabla B$ drift is 
obtained either in the usual lower single null configuration with "reversed" $B_{T}$ polarity, here referred to as (LSNrev), or in the upper single null (USN) with the usual $B_{T}$ polarity. The possibility of reversing $B_{T}$ only (not plasma current) in LSN, used in our older experiments, is not possible any longer since 1996. In fact, for the LSNrev, the plasma current must also be reversed and the NBI is then in the counter direction which causes strong impurity production, high radiative power and non-steady discharges. This is inadequate for confinement purposes and the I-mode studies in this configuration were carried out with ECRH as main heating. The advantage of LSNrev is a good pumping capability and therefore access to lower density than in USN as there are no pumps in the upper divertor. Thus, comparison between the two configurations can only be made at medium and high density. I-modes could be produced indifferently with NBI, ECRH or ICRH, whereas the discharges presented here were carried out with NBI or ECRH, or a combination of the two. The results reported in this article have been obtained during the last years and are based on the following series of I-mode experiments in deuterium with the unfavourable ion $\nabla B$ drift:

- 2009 campaign: a couple of discharges in the LSNrev configuration with ECRH which demonstrated the possibility of achieving I-modes with this heating method. This was the restart of I-mode studies at ASDEX Upgrade;

- 2011 campaign: several discharges in USN with NBI, ECRH and ICRH to investigate the access and confinement properties of the I-mode. In addition, a few discharges in the LSNrev configuration with ECRH were carried out which extend the operational window to lower densities. The charge exchange diagnostics were extended and upgraded (see below);

- 2012-2013 campaign: I-mode discharges in USN in which density fluctuations and radial electric field in the plasma edge were measured with the Doppler reflectometry and charge exchange diagnostics. Some discharges in LSNrev were conducted for the I-mode identity comparison with Alcator C-Mod as a contribution to the ITPA Joint Experiment TC-18, [16].

The plasma facing components were tungsten in all the discharges presented here, while the original Improved L-mode studies were carried out with the carbon wall. This indicates that the I-mode can be obtained independently of the plasma facing component material. 
ASDEX Upgrade is equipped with all standard diagnostics of a present-day tokamak. In this paragraph we provide information on the diagnostics which are essential for our results. The electron density is measured in the plasma core with a five channel DCN interferometer. In the following we present time traces from the DCN line-averaged densities, one of them is labelled "core" as it corresponds to a line of sight which passes close to the plasma axis, while the other is labelled "edge" and represents a line of sight whose tangency radius is generally close to the $\rho_{\text {pol }} \approx 0.85$ flux surface, the diagnostic and geometry details are provided in [17]. The edge density measurement from the lithium beam diagnostic, [18], is combined with the interferometer in the Integrated Data Analysis (IDA) to provide a full density profile, $n_{e},[19]$. The electron temperature measurement is provided by a 60 channel electron cyclotron emission heterodyne radiometer (ECE diagnostic). As well-known, the ECE diagnostic delivers the radiation temperature, $T_{\text {rad }}$, which is not necessarily the actual local electron temperature, $T_{e}$, if the optical thickness is not high enough. Large differences between $T_{\text {rad }}$ and $T_{e}$, whereby $T_{\text {rad }}$ is larger than $T_{e}$, arise in particular at the edge of the plasma where the gradients are large and the optical depth no sufficient. For this reason, a method based on the forward modelling of the ECE radiation transport has been developed and implemented in the IDA framework which provides, also at the plasma edge, the actual electron temperature deduced from the ECE signals, as described in detail in [20]. In this analysis, the electron temperature data from the ECE diagnostic and the density data from the Li-beam are treated in a combined way yielding consistent and reliable profiles for $T_{e}$ and $n_{e}$, consequently also for the electron pressure, $p_{e}$. The example illustrated in figure 1 shows profiles of $T_{\text {rad }}$ (ECE points) and $T_{e}$ (lines) at the plasma edge for different phases of a discharges which will be discussed later: low power L-mode, L-mode with higher power and following I-mode at the same input power. At low temperature the difference between $T_{\text {rad }}$ and $T_{e}$ is small but it clearly increases as the temperature increases and the difference between $T_{\text {rad }}$ and $T_{e}$ at the plasma edge becomes significant for $\rho_{p o l}>0.97$ such that the actual $T_{e}$ edge gradient is much steeper than that derived from $T_{r a d}$. The electron temperature yielded by this method will be used throughout this paper and the $T_{e}$ profiles will always be represented by lines which result from the IDA analysis and not by the ECE data points. Similarly, we will also use the IDA $n_{e}$ and $p_{e}$ data.

The ion temperature, $T_{i}$, and plasma rotation are measured with the CXRS diagnostic using NBI beam Nr. 3 as neutral source. The capabilities of these measurements have been 


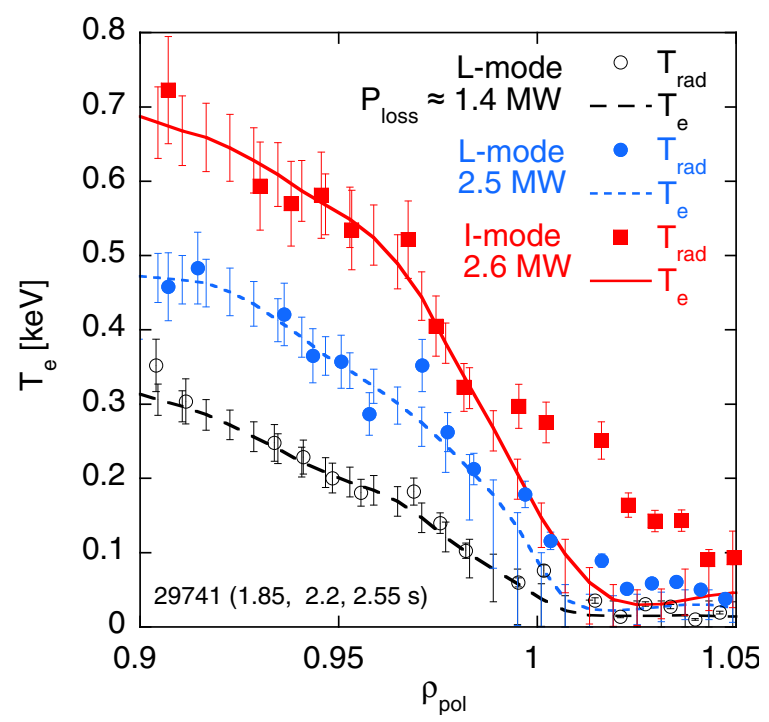

Figure 1. Comparison of the radiation temperature from ECE and the actual thermal temperature provided by the IDA analysis for three phases in the same discharge: low power L-mode, L and I modes with almost the same power.

significantly improved since 2011 by an upgrade of the core system, which has a toroidal viewing geometry, [21], and by the installation of two edge systems with viewing cords in the toroidal and poloidal directions, [22]. The upgrade of the core system provides $T_{i}$ with a $4 \mathrm{~ms}$ time resolution on 25 radial channels whereas the edge systems have a time resolution of 1.9 ms and eight radial channels each. Therefore, complete $T_{i}$ and toroidal rotation profiles, $\mathrm{v}_{\text {tor }}$, can be measured with a high time resolution. In addition, at the edge the poloidal rotation, $\mathrm{v}_{\mathrm{pol}}$, is also measured. In discharges without NBI, e.g. heated by ECRH only, $T_{i}$ and rotation can be measured using short NBI blips yielding excellent data, as described in [21].

It has been known for a long time that the edge radial electric field profile can be deduced from the ion force balance, [23, 24], using the data from the CXRS diagnostics, see [25] for ASDEX Upgrade and references therein for other devices. For ion species I the equation reads:

$$
E_{r}=\frac{1}{n_{I} Z_{I} e} \frac{\partial p_{I}}{\partial r}-\mathrm{v}_{\mathrm{pol}, \mathrm{I}} \mathrm{B}_{\text {tor }}+\mathrm{v}_{\text {tor }, \mathrm{I}} \mathrm{B}_{\mathrm{pol}}
$$

where $p_{I}$ is the pressure, $n_{I}$ the density, $Z_{I}$ the ion charge and $e$ is the elementary charge. In the last two terms $v_{x} B_{y}$ are the ion velocities and magnetic fields in the poloidal or toroidal directions. The first term is often named diamagnetic contribution.

Due to the limited number of radial points of the edge CXRS diagnostic until the end of the 
2013 campaign, the obtention of a full $E_{r}$ profile required a shift of the radial plasma position of about $1 \mathrm{~cm}$ which lasts about $200 \mathrm{~ms}$, if the NBI is turned on continuously. In ECRH heated discharges the measurement is only possible during the NBI blips and the time resolution is much longer as several blips separated by at least $100 \mathrm{~ms}$ each are required for the same plasma conditions whereas the outer position of the plasma is scanned. The $E_{r}$ profile deduced from this diagnostic is named $E_{r}^{C X R S}$ and is available since 2009. A first $E_{r}^{C X R S}$ profile for I-mode in ASDEX Upgrade was reported in [25].

The characteristic instabilities of the I-Mode, WCM and GAM, are, as in Alcator CMod, measured in ASDEX Upgrade with reflectometry and magnetic probes. Temperature fluctuations are not measured. In addition to the usual reflectometers, [26, 27], so-called Doppler reflectometers are also available at ASDEX Upgrade which provide the perpendicular rotation of the plasma from which the radial electric field can be deduced, [28, 29]. We label this quantity $E_{r}^{D R}$. These profiles agree generally with those deduced from the CXRS diagnostic within the uncertainties of the two diagnostics, see [25] for ASDEX Upgrade. For the measurement of the $E_{r}$ profile, the Doppler reflectometry method requires a frequency scan of the probing micro-wave beam which takes about 100ms, which limits the time resolution. The L-I transition can be identified by the increase of the edge temperature, whereby $T_{e}$ is better suited than $T_{i}$, and by the appearance of the WCM and GAM. The confinement time also follows which confirms the identification of the transition. The reflectometer was not measuring in all the older discharges such that the occurrence of the WCM is not always available, but the other criteria are sufficient to identify the L-I transition. The accuracy of determination of the L-I transition in time can sometimes reach $\pm 50 \mathrm{~ms}$ which, however, is sufficiently short for the analyses presented below because the discharges vary slowly around the L-I transition.

Finally, throughout this paper, we characterise the total heating power by the usual loss power defined as $P_{\text {loss }}=P_{\text {heat }}-d W / d t$, where $P_{\text {heat }}$ is the sum of all the heating powers taking into account the respective absorptions and fast ion losses for NBI, while W is the plasma energy. For the energy confinement time we use the thermal confinement time whereby the fast ion contribution to the plasma energy is subtracted. This quantity is used to calculate the $\mathrm{H}$ mode enhancement factor $H_{98 y, 2}$ according to the commonly used ITER confinement scaling IPB98(y,2), [30]. For simplicity we write $H_{98}$ throughout the paper. 


\section{I-mode main properties: NBI and ECRH comparison}

In the following we illustrate by a few examples the main properties of the I-modes obtained in the configurations LSNrev and USN, which both have the ion $\nabla B$ drift away from the X-point.

\subsection{I-mode with ECRH}

The first example deals with two ECRH-heated discharges from 2009 in LSNrev configuration with the same controlled parameters but developing the I-mode in different ways. These were carried out to explore, for the first time as proof of principle, the possibilities to obtain the I-mode with ECRH. The absolute values of plasma current $\left(I_{p}=1 \mathrm{MA}\right)$ and magnetic field $\left(B_{T}=2.5 \mathrm{~T}\right)$, yielding an edge safety factor $q_{95} \approx 4.5$, are in the usual range for ASDEX Upgrade. The line-averaged density, $\bar{n}_{e} \approx 4 \times 10^{19} \mathrm{~m}^{-3}$, is in the medium range for the device. A few time traces for each discharge are shown in figure 2.

The ECRH power is increased in three steps up to about $2.2 \mathrm{MW}$ and then decreased, using exactly the same timing and power for the two discharges, as shown in panel (a). During the first ECRH power step (1.5 - $2.0 \mathrm{~s}$ ) the confinement factor $H_{98}$ (panel e) remains constant at about 0.45 , as expected in the L-mode. During the second ECRH step, $2.0-3.0 \mathrm{~s}$, the two discharges behave differently. While shot 25875 is in steady state after the initial changes following the ECRH power step, shot 25874 exhibits a gradual confinement improvement, which starts at about $2.3 \mathrm{~s}$ as reflected by an increase of the edge electron temperature (panel c), plasma energy (panel d) and $H_{98}$ factor (panel e). The latter reaches about 0.75 while the other discharge remains at $H_{98} \approx 0.5$. Discharge 25874 goes into the I-mode whereby the L-I transition takes place at about $2.3 \mathrm{~s}$ with $P_{\text {loss }} \approx 2 \mathrm{MW}$. The slow evolution is typical of the I-mode evolution in ASDEX Upgrade. The confinement improvement develops gradually over $\approx 300 \mathrm{~ms}$ which corresponds to about three confinement times. Due to the slow change, the time point at which this evolution starts cannot be determined with very high accuracy: in contrast to the L-H transition, the L-I transition is not abrupt and determining the time at which it occurs and the corresponding parameters suffer from uncertainties. In the present case, due to the lack of reflectometry measurement the WCM cannot be used to identify the L-I transition but the appearance of the GAM at 2.3s is consistent with the increase of the edge $T_{e}$. However, if, as in the case of 25874, the L-I transition occurs in phases 


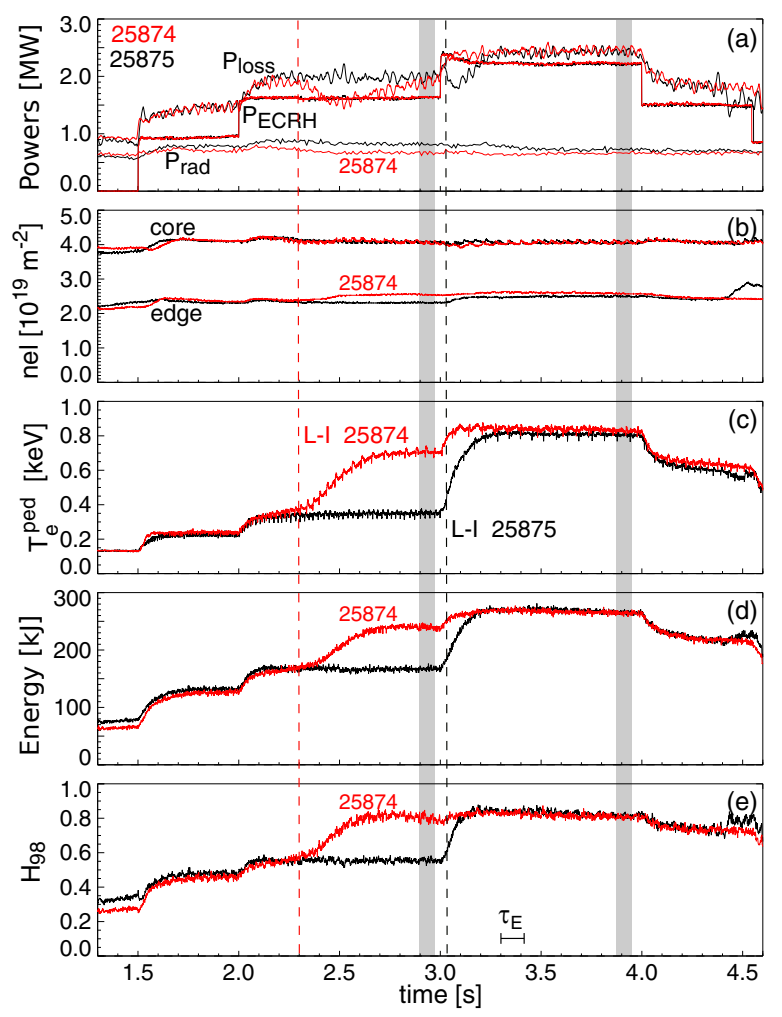

Figure 2. Comparison of two I-mode discharges with ECRH in the LSNrev configuration: panel (a): $P_{E C R H}, P_{\text {loss }}$ and main plasma radiative power; $(b)$ core and edge line-integrated densities; (c) pedestal $T_{e}$ at $\rho_{\text {pol }} \approx 0.97$; (d) plasma energy; (e) enhancement factor $H_{98}$. The vertical dashed lines indicate the respective L-I transitions, the grey bars the time interval for the profile analysis, plotted in the next three figures.

during which the parameters such as power and density do not change quickly, the plasma parameters representative for the L-I transition can be obtained with a sufficient accuracy to be meaningful, see sections 4 and 5. A further observation is that both core and edge densities do not change for discharge 25875 which remains in the L-mode, while, in discharge 25874 , the edge density increases by about 5\% after the L-I transition, showing that particle transport is not completely insensitive to the L-I transition. The time constant of the density increase is similar to that of the pedestal temperature.

The edge density profiles for $\mathrm{L}$ and I mode at about $2.9 \mathrm{~s}$ are shown in figure 3 . With the occurrence of the I-mode the density increases slightly in the radial region $0.85<\rho_{\text {pol }}<0.98$. It is unlikely that this change is due to an increase of the particle source because the lineaveraged core density is feedback-controlled such that the gas fuelling is reduced as the 


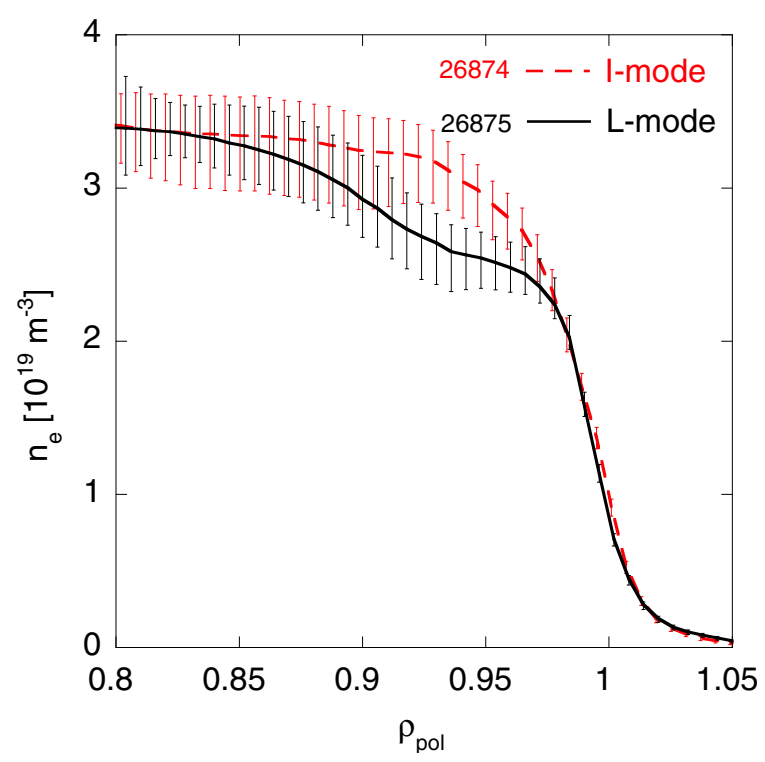

Figure 3. Density profile in the pedestal region for I-mode and L-mode taken at $2.9 \mathrm{~s}$ in discharges 25874 and 25875 respectively.

density starts to increase after the L-I transition. After about $100 \mathrm{~ms}$ the gas flux through the valve has been reduced by about $50 \%$ and remains at this lower level. In the L-mode discharge the fuelling is constant until the next ERCH step at 3.0s. Therefore, at least in the plasma edge, a slight reduction of particle transport probably occurs after the L-I transition. Whether this is due to a reduction of the particle diffusion or an increase of the pinch cannot be determined. It should be underlined, however, that the typical signatures of a transition to $\mathrm{H}$-mode are not detected in the usual monitors, such as $\mathrm{D}_{\alpha}$ in divertor, indicating that the transport reduction is indeed small and slow.

The fact that discharge 25875 does not transition to I-mode during the second ECRH step is attributed to the somewhat higher radiation losses (see panel a) caused by a small impurity event at the end of the current ramp-up. It will be shown in section 5 that during the second ECRH power step these shots were very close to the L-I power threshold which is $P_{\text {loss }} \approx 2$ MW at $\bar{n}_{e} \approx 4 \times 10^{19} \mathrm{~m}^{-3}$. However, discharge 25875 goes into I-mode when the third ECRH power step is turned on, as clearly indicated by the stronger increase of temperature, plasma energy and $H_{98}$ compared to 25874 . The time of the L-I transition for shot 25875 is identified by the appearance of the GAM at about $3.03 \mathrm{~s}, 30 \mathrm{~ms}$ after the start of the third ECRH step. This is consistent with the hypothesis that the higher radiation losses of about $100 \mathrm{~kW}$ should be compensated by somewhat more heating power. During the steady-state phase of the third 
ECRH step, both discharges exhibit exactly the same values, including edge density. At this somewhat higher heating power the I-mode reaches $H_{98} \approx 0.8$. Thus, it should be noted that in both $\mathrm{L}$ and I mode $H_{98}$ increases with heating power. Finally, it is worth underlying that after the reduction of the ECRH power back to $1.5 \mathrm{MW}$ at $4 \mathrm{~s}$, both discharges remain in the I-mode at the confinement level of $H_{98} \approx 0.7$ At this time the radiation power is the same in the two discharges. This phase is terminated by the reduction of the ECRH power at $4.55 \mathrm{~s}$ and the ramp down of the discharges which starts at 4.6s. Therefore the I-mode of discharge 26874 was maintained with $H_{98} \geq 0.7$ during more than 2 s, which corresponds to about 20 confinement times. With $H_{98} \approx 0.8$ during the highest power step, the confinement time is significantly above the L-mode level, but somewhat below that of the H-mode, as will be shown quantitatively in comparison with other discharges in a next section. In contrast to other discharges which will be discussed below, no transition from I to $\mathrm{H}$ mode occurred in these discharges because the heating power was not sufficient to induce it.

As these discharges are from 2009, before the upgrade of the CXRS system and with ECRH only, no $T_{i}$ measurement is available, but this pair offers during the second ECRH power step a nice and rare comparison of the $T_{e}$ profiles in $\mathrm{L}$ and I mode at almost the same heating power over a few confinement times. The $T_{e}$ core profiles measured at the end of the second ECRH power step (2.9s), I-mode for 25874 and L-mode for 25875, as well as those taken at the end of time interval with maximum ECRH power (3.9s), both in I-mode, are shown in figure 4. The time intervals are indicated by grey bars in figure 2. Of course the core temperatures increase with the ECRH power, but a main contribution is caused by the formation of the I-mode pedestal.

The electron temperature pedestal is illustrated in more detail by a zoom in the edge region presented in figure 5 . With its top at $\rho_{\text {pol }} \approx 0.97$, it is similar to that observed in our older discharges, [5], and to the Alcator C-Mod results, e.g. [6, 8]. The temperature at the top of the pedestal is significantly higher than in the L-mode and it increases with ECRH power, which corresponds to an increase of the pedestal pressure gradient.

As some diagnostics, in particular the $T_{i}$ measurement, were not available at the time of these experiments we do not discuss them in more detail. A more complete analysis of the I-mode properties and a comparison between NBI and ECRH heated I-modes is provided in the next sections. 


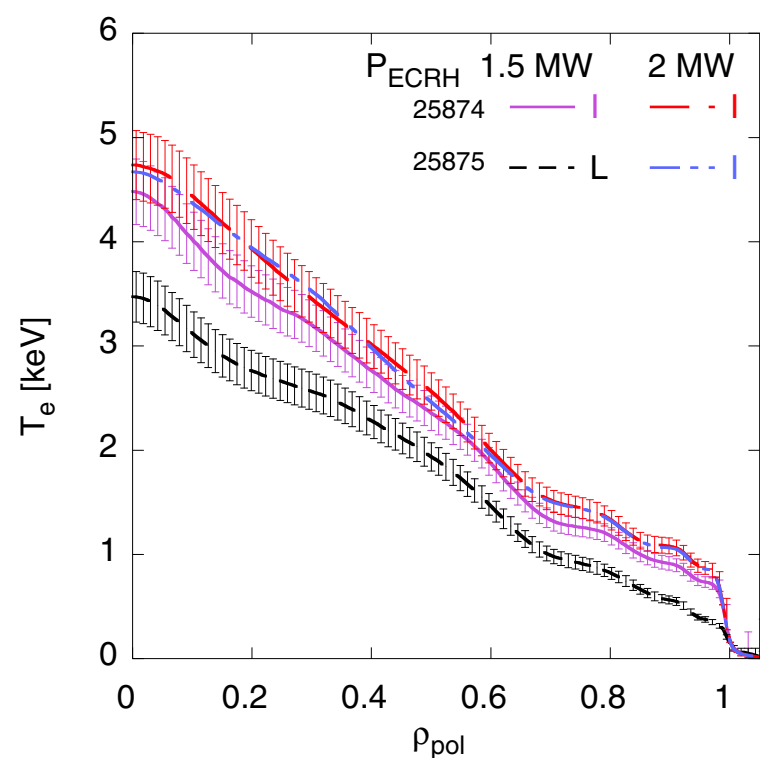

Figure 4. Comparison of $L$ and I mode core $T_{e}$ profiles for discharges 25874 and 25875, taken at $t=2.9$ s and 3.9s, see Fig. 2.

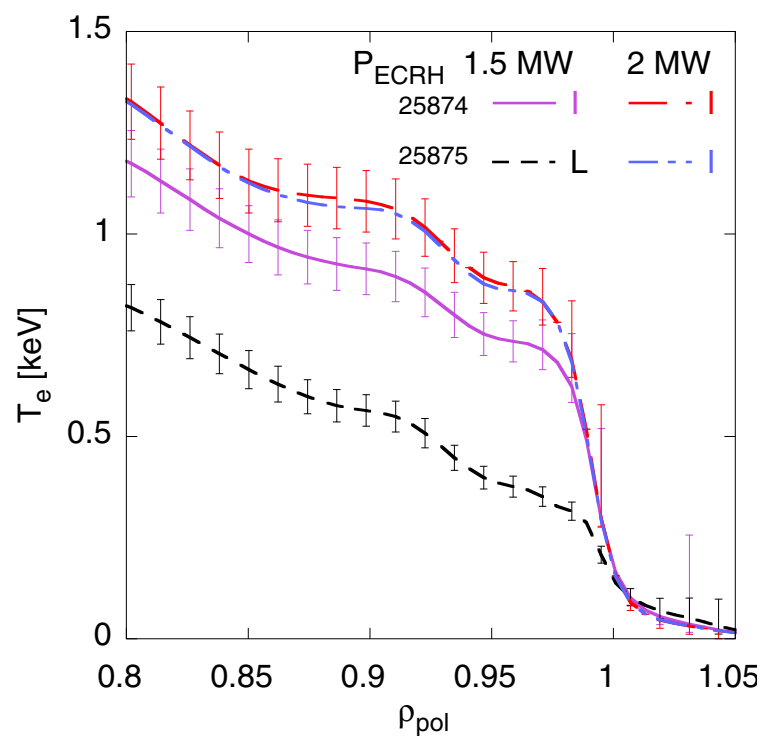

Figure 5. Zoom of the edge region of figure 4: comparison of $L$ and I mode pedestal $T_{e}$ profiles for discharges 25874 and 25875, taken at $t=2.9$ s and 3.9s, see Fig. 2.

\subsection{Comparison of I-modes induced by ECRH or NBI}

During the 2011-2013 campaigns we investigated I-modes produced with ECRH and NBI in the USN configuration. As indicated above, complete profiles of $T_{i}$ were provided by the core and edge CXRS diagnostics, whereby NBI blips were used for the ECRH heated discharges. 
Two examples of such discharges are shown in figure 6 where the left panels show the NBI case and the right ones that with ECRH.
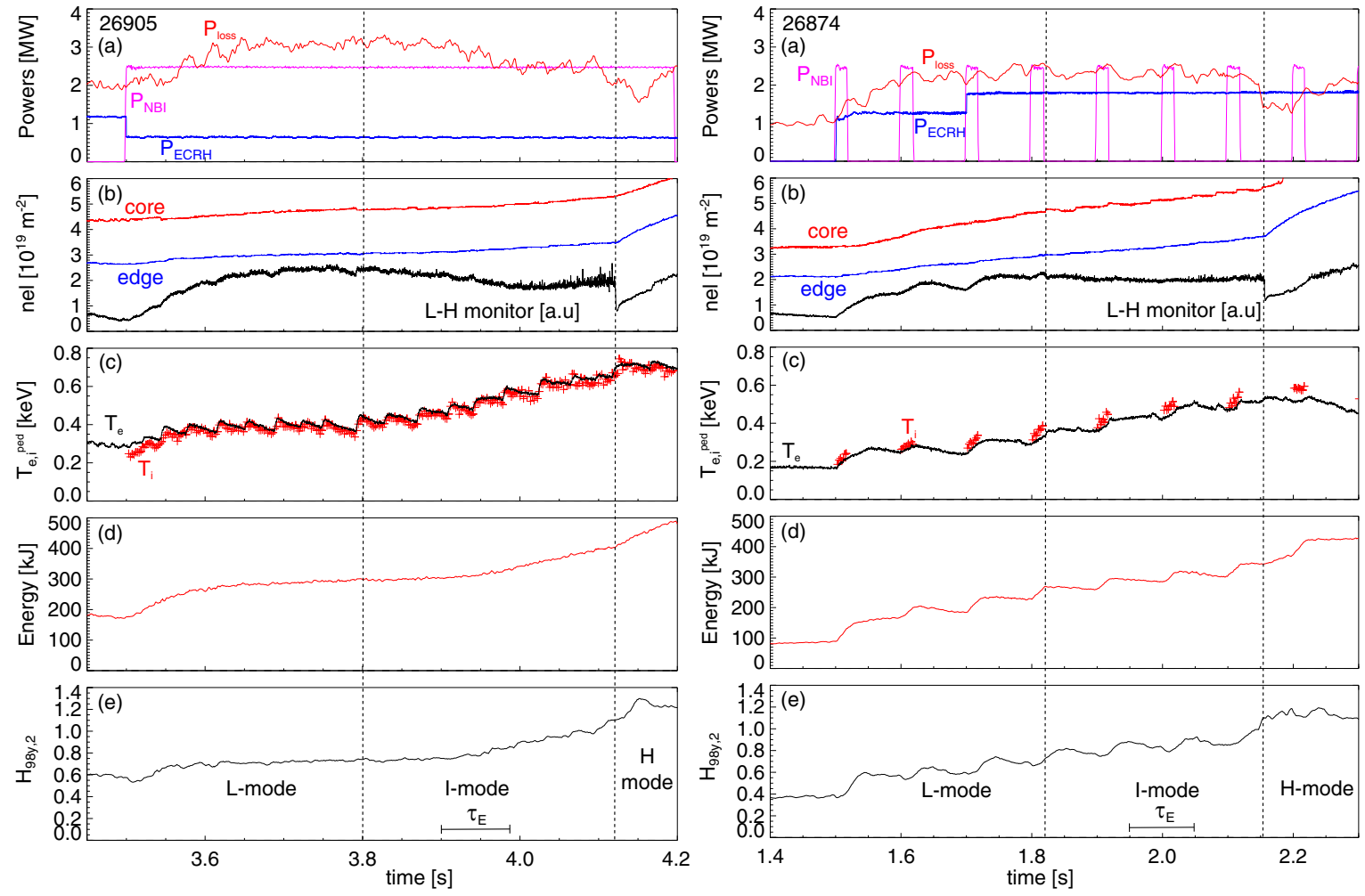

Figure 6. Time traces of two I-mode discharges NBI (left) and ECRH (right) in USN configuration, campaign 2011. Panel (a): heating powers; Panel (b) core and edge interferometry channels and ELM and L-H monitor; Panel (c): edge $T_{e}$ and $T_{i}$ taken at $\rho_{\text {pol }}=0.97$; Panel (d) plasma energy; Panel (e) normalised confinement time $H_{98}$.

The panels show time traces of the same quantities for each discharge. In both cases the auxiliary heating power is constant before the L-I transition and during the I-mode. Both discharges went from $\mathrm{I}$ to $\mathrm{H}$ mode at constant input power, an interesting feature discussed in more detail below. The times of the L-I and I-H transitions are indicated in the figure by vertical lines. The NBI-heated case is discharge 26905 illustrated in the left column of plots. The plasma is heated with beam Nr. 3, delivering 2.5 MW and allowing $T_{i}$ to be measured continuously in time. The ECRH power of about $0.6 \mathrm{MW}$ is turned on before the NBI to prevent possible tungsten accumulation. The heating power $P_{l o s s}$ is then about $3 \mathrm{MW}$. An inwards-outwards scan of the outer plasma position by about $1 \mathrm{~cm}$ was performed between $3.8 \mathrm{~s}$ and $4.1 \mathrm{~s}$ to increase the radial coverage and resolution of the edge CXRS system, which yielded the $E_{r}$ profile for the I-mode reported in [25]. 
The ECRH-heated example is discharge 26874, illustrated in the right plot column. It has two steps of ECRH power, 1.3 and $1.8 \mathrm{MW}$, yielding $P_{\text {loss }} \approx 2.3 \mathrm{MW}$ during the second ECRH step, which is somewhat lower than in the discharge with NBI. We applied NBI blips from beam Nr. 3 for the CXRS measurements.

In contrast to the discharges discussed in the previous subsection, the I-mode in these two discharges is not stationary and ends in both cases with a transition to H-mode, clearly indicated by the sudden increase of the edge density and the sharp drop of the L-H monitor (see panels b). The L-H monitor is one channel of the AXUV diode bolometer cameras, [31], which looks at the USN X-point region. Its geometry is better suited than our $D_{\alpha}$ measurement in the upper part of the machine. This H-mode is ELM-free because the heating power is close to the power threshold. As we already pointed out in our previous work, [5], the I-H transition exhibits the following features: the transition, not preceded by dithering or I-phase, is abrupt as indicated by the fast drop in the $\mathrm{D}_{\alpha}$ and AXUV signals, as well as by the strong edge density increase. The absence of I-phase before the I-H transition seems to be very general but not necessarily universal. In contrast, I-phases are observed at the end of the H-mode, as also reported in [11]. It should be underlined that the increase of the pedestal temperature at the I-H transition is very weak, in agreement with the fact that the temperature pedestal was already formed during the I-mode and also because the density increases strongly. Further, it is worth noticing that no type-III ELMs occur after the I-H transition which is attributed to the low collisionality induced by the I-mode pedestal. This is not necessarily a general feature, but observed very often in our study and is coherent with the absence of I-phase. The increase of $H_{98}$ after the I-H transition is small, in agreement with the fact that its value is already close to unity before the I-H transition.

Coming back to the I-mode in these two discharges: After the L-I transition, the I-mode temperature pedestal develops (panel c), leading to an improvement of global confinement, as reflected by the plasma energy and $H_{98}$ factor (panels d and e). Just before the I-H transition $H_{98}$ reaches about 1 in both cases. It must be underlined that the evolution of the I-mode occurs over about three confinement times and is therefore slow. It is also important to note that the I-mode temperature pedestal builds up similarly for both $T_{e}$ and $T_{i}$, which remain equal as shown in panels c for the pedestal values and will be further documented below by the profiles. It is also essential to underline that the enhancement of the pedestal occurs while $P_{\text {loss }}$ decreases which is due to the $d W / d t$ contribution and, to a lesser extent, to a 
slight decrease of the Ohmic power. Therefore, the I-H transition occurs at values of $P_{\text {loss }}$ which are lower than that of the L-I transition. The density also increases slowly which is mainly due to the poor pumping in the USN configuration, whereby a slight increase of particle confinement is not excluded, as pointed out above. Further features should be emphasised. The fluctuations visible on the L-H monitor (in particular for discharge 26905) which becomes strong about $100 \mathrm{~ms}$ before the I-H transition, were also observed in our earlier experiments, [5], and will be discussed in a next section. The low frequency oscillations on the pedestal temperatures for discharge 26905 are caused by the sawtooth heat pulses. There are indications that the sawtooth pulse at about $3.8 \mathrm{~s}$ triggers the L-I transition for discharge 26905, a well-known effect for the L-H transition and also reported for the L-I transition in Alcator C-Mod, [8, 9, 13]. For the ECRH-heated discharge the L-I transition seems to be induced by the NBI blip at $1.8 \mathrm{~s}$.
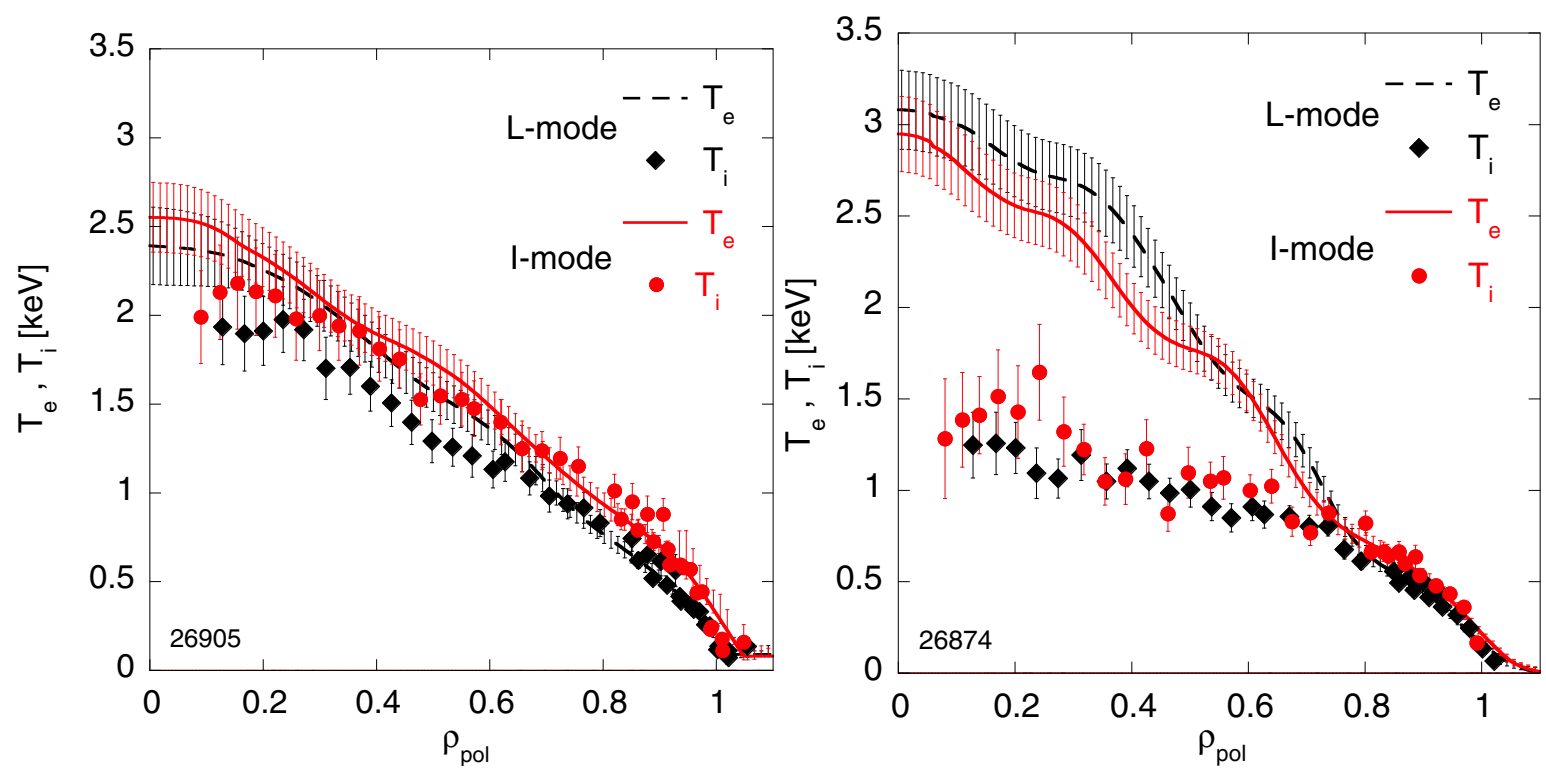

Figure 7. Comparison of the $T_{e}$ and $T_{i}$ profiles, taken just before the L-I and I-H transitions for discharges 26905 (NBI) and 26874 (ECRH). The transition time points are indicated in Figure 6.

In the following we compare the temperature profiles for these two discharges, starting with the core temperatures in figure 7, left the NBI-heated case, right the ECRH case. With NBI heating the $T_{e}$ and $T_{i}$ profiles are close to each other over the whole radius. In contrast, with ECRH, due to the dominant electron heating, $T_{e}$ is much higher than $T_{i}$ in the plasma core, with central $T_{e}$ of about $3 \mathrm{keV}$ and $1.5 \mathrm{keV}$ for $T_{i}$. These profiles, including the quite 
flat $T_{i}$, are typical for ECRH-heated ASDEX Upgrade plasmas, see e.g. [21]. Due to the collisional energy exchange, in this case from the electron to the ion heat channel, the $T_{e}$ and $T_{i}$ profiles converge towards the same values at the plasma edge, such that the pedestal values are comparable in the NBI and ECRH cases. As compared to the 2009 I-mode with ECRH (previous sub-section), the increase of the core temperatures from L to I mode is smaller which is attributed to the fact that the density increases during the I-mode.

The surface-integrated electron and ion heat fluxes, respectively $Q_{e}$ and $Q_{i}$, deduced from the power balance analysis taken at the L-I transition for these two discharges are shown in figure 8. The temperature profiles plotted in figure 7 are used and yield the lines for $Q_{e}$ and $Q_{i}$. The error bars are mainly due to the uncertainties on the electron-ion collisional energy transfer and significantly increase towards the edge due to the strong increase of the collisionality and to the reduction of the difference $T_{e}-T_{i}$ with respect to the experimental uncertainties on the temperatures. They become very large for $\rho_{\text {pol }}>0.9$ where the analysis is then meaningless and we did plot the results in this part of the plasma. As expected, in the central of the plasma, the electron and ion heat fluxes for the two discharges are very different: much higher electron heat flux and lower ion heat flux for the ECRH-heated discharge. However, due to the collisional energy exchange which plays a key role in the ECRH-heated discharge, $Q_{e}$, but in particular $Q_{i}$ converge respectively towards the same value at the plasma edge in these two discharges. In other words, at the radial position of the top of the pedestal which forms at the time of the L-I transition the ion heat fluxes are the same independently of the heating method. This probably explains the similarities between the NBI and ECRH heated discharges in this density range. This can be very different at lower density where the heat channels are much less coupled, even at the plasma edge, as discussed in a next section.

We now compare in figure 9 the pedestal temperature profiles in L, I and $\mathrm{H}$ modes, for NBI on the left and for ECRH on the right. The $T_{e}$ and $T_{i}$ profiles are indeed very similar in the edge, independently of the heating method. No temperature pedestal can be recognised in the L-mode, but it appears clearly in the I-mode which is a characteristic of this regime. The pedestal top is here also at $\rho_{p o l} \approx 0.97$, for both $T_{e}$ and $T_{i}$. We remind here that $T_{e}$ (lines in the plots) is provided by the IDA procedure described in section 2 . As already mentioned, the temperature pedestal in the $\mathrm{H}$-mode is only slightly above the value reached before the I-H transition. The gradient of $T_{e}$ seems to steepen somewhat in the H-mode, which suggests a further enhancement of the edge transport barrier for the heat. A similar effect for $T_{i}$ cannot be 


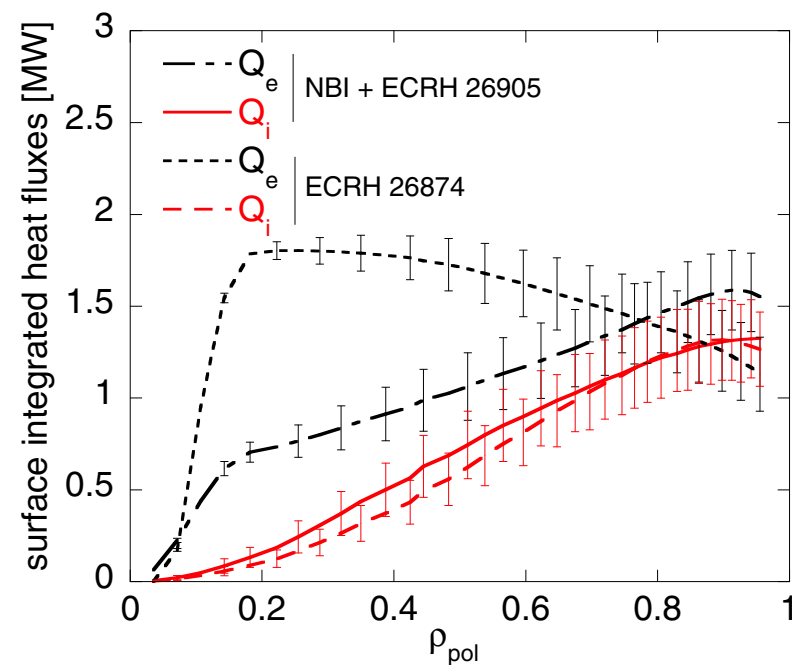

Figure 8. Comparison of the surface-integrated heat fluxes for the electron and ion channels taken just before the L-I transition for discharges 26905 (NBI) and 26874 (ECRH). The transition time points are indicated in Figure 6.
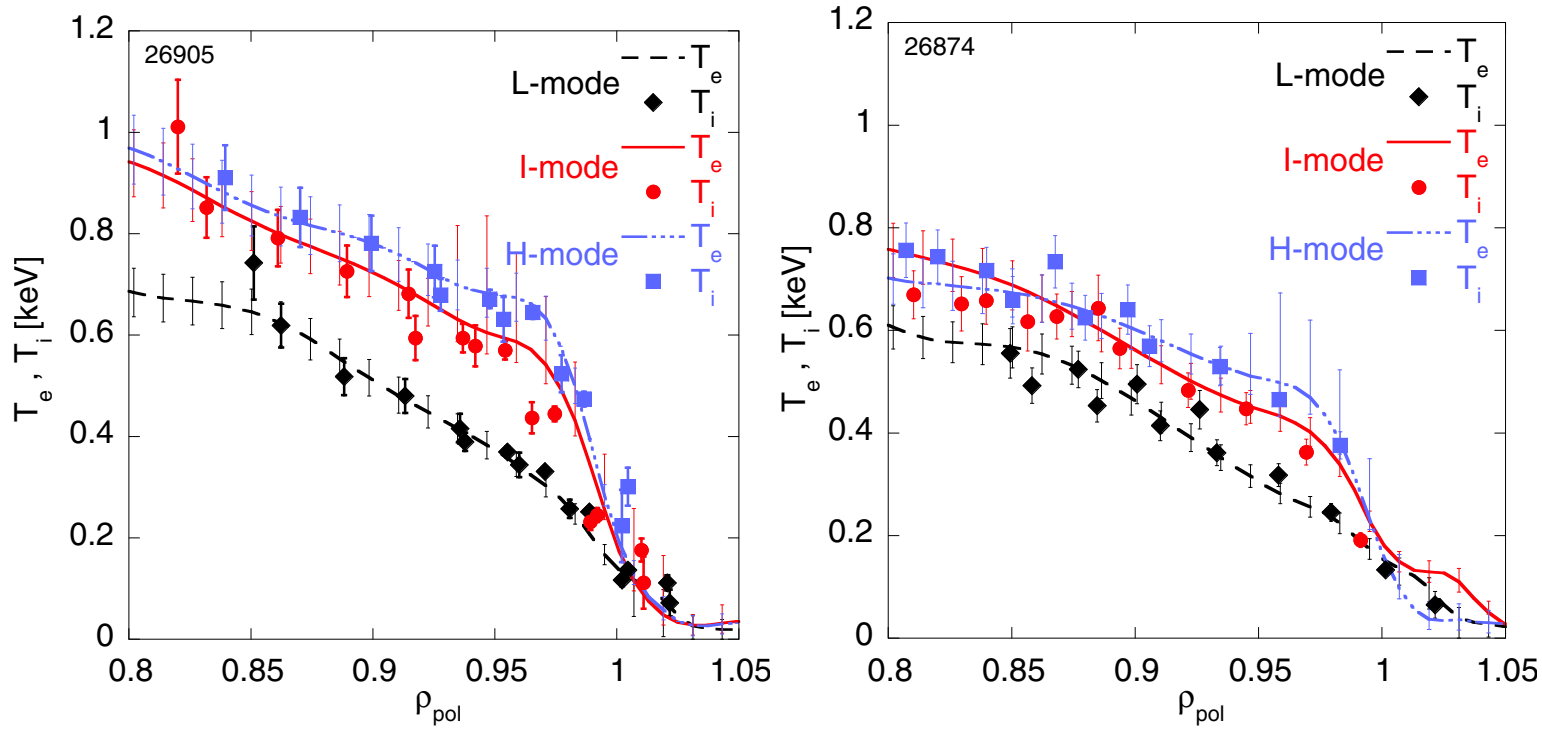

Figure 9. Profiles of $T_{e}$ (lines) and $T_{i}$ (data points) in the pedestal region for discharges 26905 (NBI, left panel) and 26874 (ECRH, right panel). For L and I modes the data are taken just before the L-I and I-H transitions. The H-mode profiles are taken 100 ms after the I-H transition.

excluded but the steep gradient region of the $T_{i}$ pedestal is not measured sufficiently well. In particular we cannot exclude either that the pedestal gradient of $T_{i}$, in the region $\rho_{\text {pol }}>0.97$, might be somewhat flatter than that of $T_{e}$, see e.g. figure 9 left panel. Due to the somewhat lower heating power in the ECRH case the pedestal top temperatures are a bit lower with 
ECRH than with NBI, but the relative changes from L to I and $\mathrm{H}$ modes are similar.
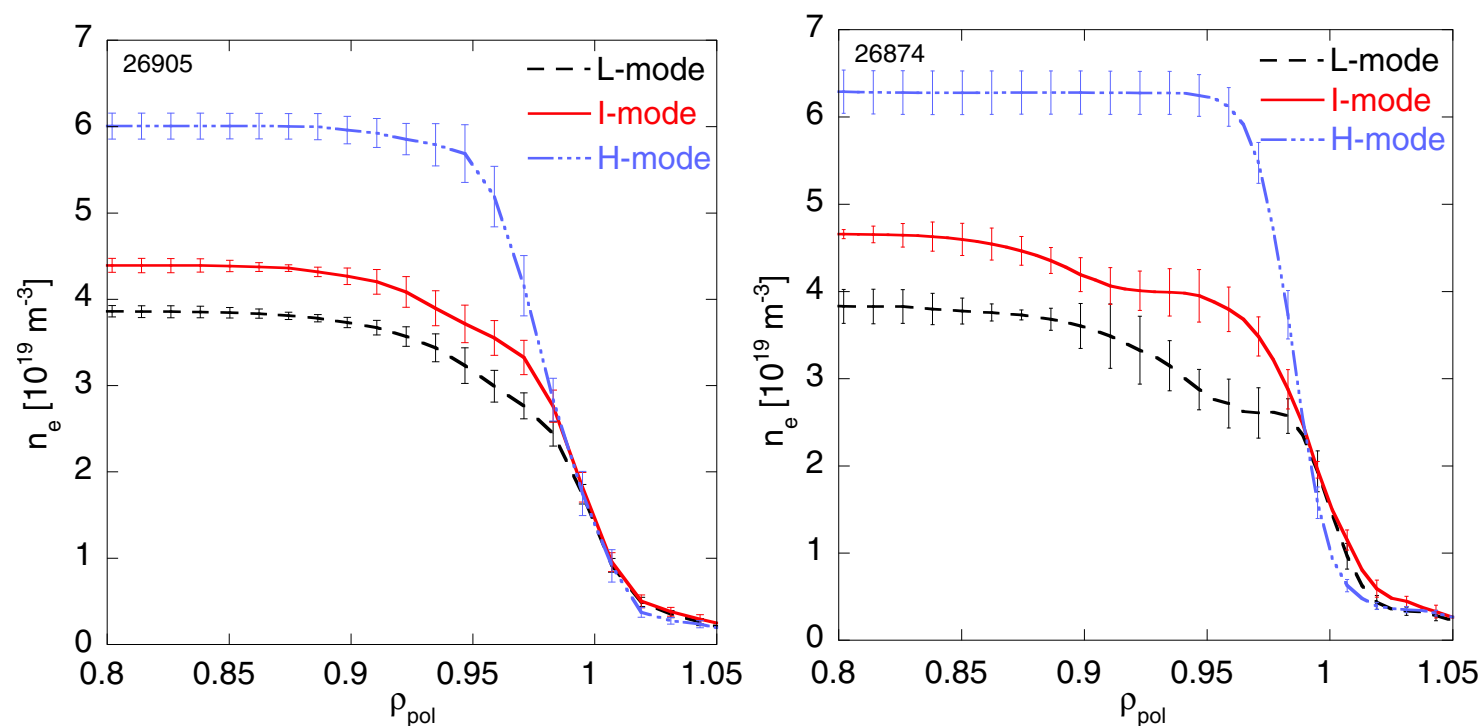

Figure 10. Density profiles in the pedestal region for discharges 26905 (NBI, left panel) and 26874 (ECRH, right panel). For L and I modes the data are taken just before the $L$-I and I-H transitions. The H-mode profiles are taken $100 \mathrm{~ms}$ after the I-H transition.

The pedestal density profiles are illustrated in figure 10. The small density increase which occurs during the I-mode is clearly seen. The pedestal shape does not change significantly between $\mathrm{L}$ and I mode, although, in the ECRH case a weak peaking around the pedestal top in the I-mode, reminiscent of the effect exhibited in figure 3, is not excluded. In contrast, after the transition to H-mode, the density pedestal is much clearer with the characteristic very steep gradient. This change of the density profile is very similar for NBI and ECRH heated discharges.

The combined effects of the changes in electron temperature and density is reflected in the electron pressure, plot in figure 11 for the pedestal region. Here also the NBI case is displayed in the left part of the figure, the ECRH one in the right one. In addition to the pressure profile, we plotted the pressure gradient in the lower panels. The pressure profile exhibits, as expected, a significant increase from L to I and from I to H mode, whereby both the pressure at the pedestal top and the edge gradient increase. The increase from I to $\mathrm{H}$ mode is mainly due to the formation of the strong density pedestal. The pressure gradient exhibits a clear well whose minimum, $\nabla p_{e, \min }$, is getting strongly more negative from L-to-I-to-H mode. The radial position of $\nabla p_{e, \min }$ and of the pedestal top exhibit a trend to be slightly shifted inwards in the evolution from $\mathrm{L}$ to $\mathrm{H}$ mode. These features are exhibited by both NBI and ECRH 
heated discharges without significant differences. The minimum of the pressure gradient will be used in the next sections.
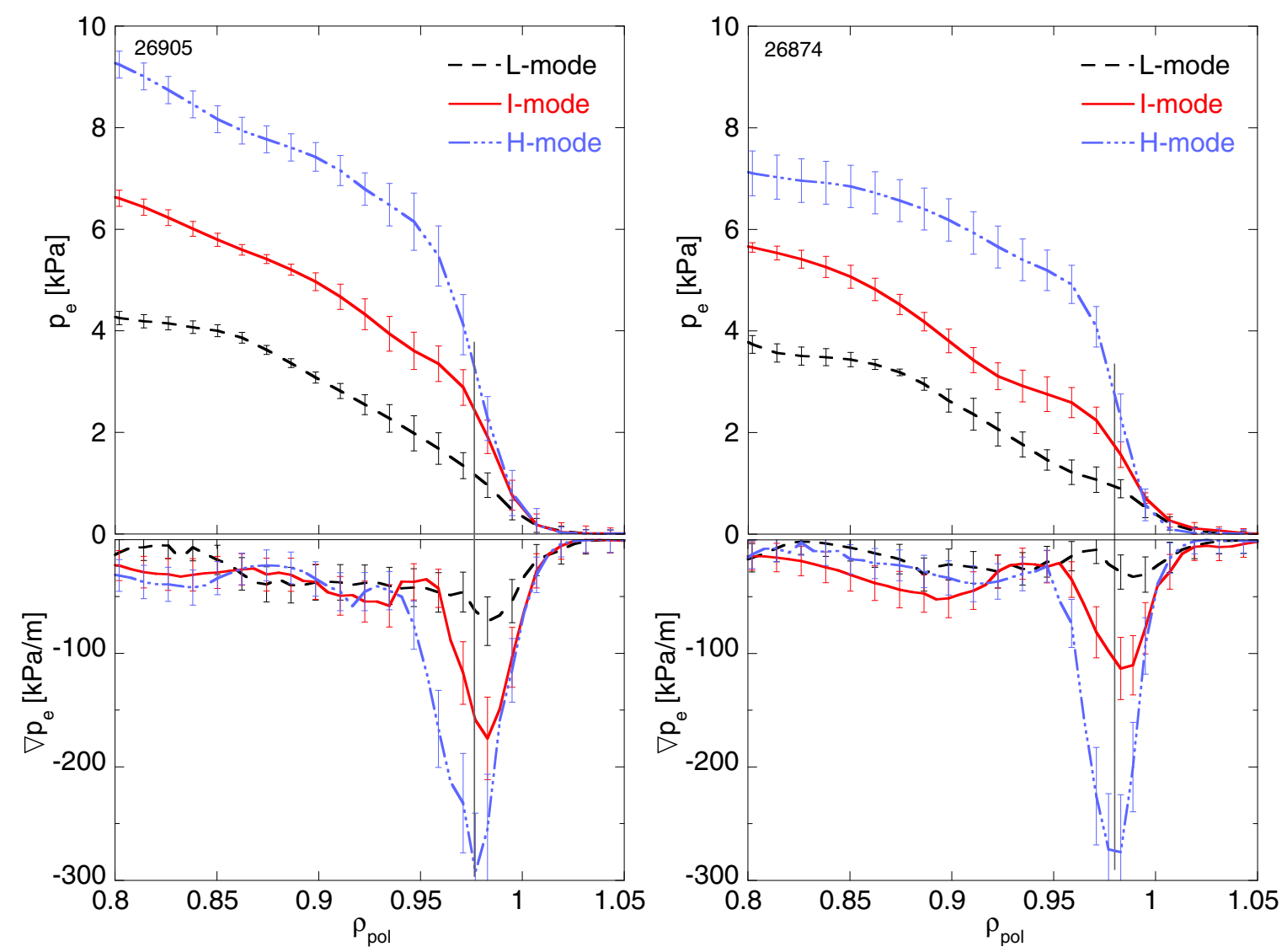

Figure 11. Edge electron pressure profiles (upper plots) and electron pressure gradient (lower plots) for the L, I, and H time points of the previous figures; left NBI case, right ECRH case. A vertical line has been added in each plot to guide the eye in relating the position of $\nabla p_{e, \min }$ to the pressure profile.

Summarising this section, we have shown that the I-mode can also be achieved with pure electron heating and compared NBI and ECRH cases. As in Alcator C-Mod, the I-mode has also been achieved with ICRH, but we do not show examples of them because they suffer from the lack of $T_{i}$ measurement. The occurrence of the I-mode does not depend on how the unfavourable magnetic configuration is created, LSNrev or USN, both yield I-modes. In all the cases, the characteristic I-mode temperature pedestal develops while the change in density is small. In the density range studied here $3.5<\bar{n}_{e}<6 \times 10^{19} \mathrm{~m}^{-3}, T_{e}$ and $T_{i}$ are equal within the error bars in the edge region, independently of the heating method. This is different at lower density where the collisional energy exchange might be too weak to bring the profiles 
together at the edge for ECRH-heated cases and a situation with $T_{e}^{\text {ped }}>>T_{i}^{\text {ped }}$ can occur, as discussed in section 5 .

\section{Fluctuations and radial electric field in the I-mode}

As indicated in the introduction, two instabilities appear at the L-I transition, WCM and GAM, $[13,14]$, and it is not clear to which extend they contribute to the transition to I-mode. In addition, it is widely accepted that the radial electric field at the plasma edge plays a key role in the formation and sustainment of the H-mode edge transport barrier through $\nabla E_{r}$ which induces a sheared $E \times B$ flow and stabilises the turbulence, [32]. The perpendicular velocity flow shear induced by $E \times B$ is proportional to $\nabla E_{r} / B$. Whether this effect contributes to the LI transition is not clarified yet and is discussed in section 5. Nevertheless, it is an experimental observation that in the narrow region at the very edge of the plasma, the $E_{r}$ profile exhibits a clear well with a negative minimum, $E_{r, m i n}$, which is often used to characterise the $E_{r}$ well, justified by the fact that the well width varies little.

The edge $E_{r}$ is essentially induced by the main ions according to neoclassical theory, [33], as assessed experimentally, see e.g. $[6,25]$. The $E_{r}$ well is weakly pronounced in L-mode, much deeper in H-modes and takes intermediate values just before the $\mathrm{L}-\mathrm{H}$ transition, [34, 35]. In the I-mode, the development of the temperature pedestal leads to a deeper edge $E_{r}$ well, as compared to the preceding L-mode and $E_{r, \min }$ lies between the L-mode and H-mode values for the corresponding plasma conditions, [6, 25, 36, 37, 38]. Finally, as shown for Alcator C-Mod in $[8,12,9]$, and also observed in ASDEX Upgrade, [14, 37, 38], the transport reduction in the I-mode pedestal is correlated with a decrease of the turbulence level in the radial region of the steep pedestal gradient. The respective contributions of $E_{r}$, WCM and GAM to the transport reduction are not clarified in the present work, but in the next two sections we report experimental observations which might contribute to the understanding.

For this purpose we discuss now a series of NBI-induced I-modes from the 2012-2013 campaign (shots 29737 - 29746). They are very similar to the NBI-heated discharge 26905 of figure 7: same plasma current, magnetic field and USN magnetic configuration, about the same density and heating power. They also exhibit the slow development of the I-mode pedestal which ends with a transition to H-mode. They differ somewhat from each other through small changes in density and heating power, which have been slightly varied on purpose to increase the duration of the I-mode. This mainly alters the time of L-I transition, 
the development of the I-mode pedestal and the time of the I-H transition. All the required measurements are available for these discharges, in particular: reflectometry to identify the WCM, Doppler reflectometry for $E_{r}$ and turbulence measurement, CXRS for $T_{i}$ and partially $E_{r}$. One of these discharges is illustrated in figure 12 by a selection of time traces.

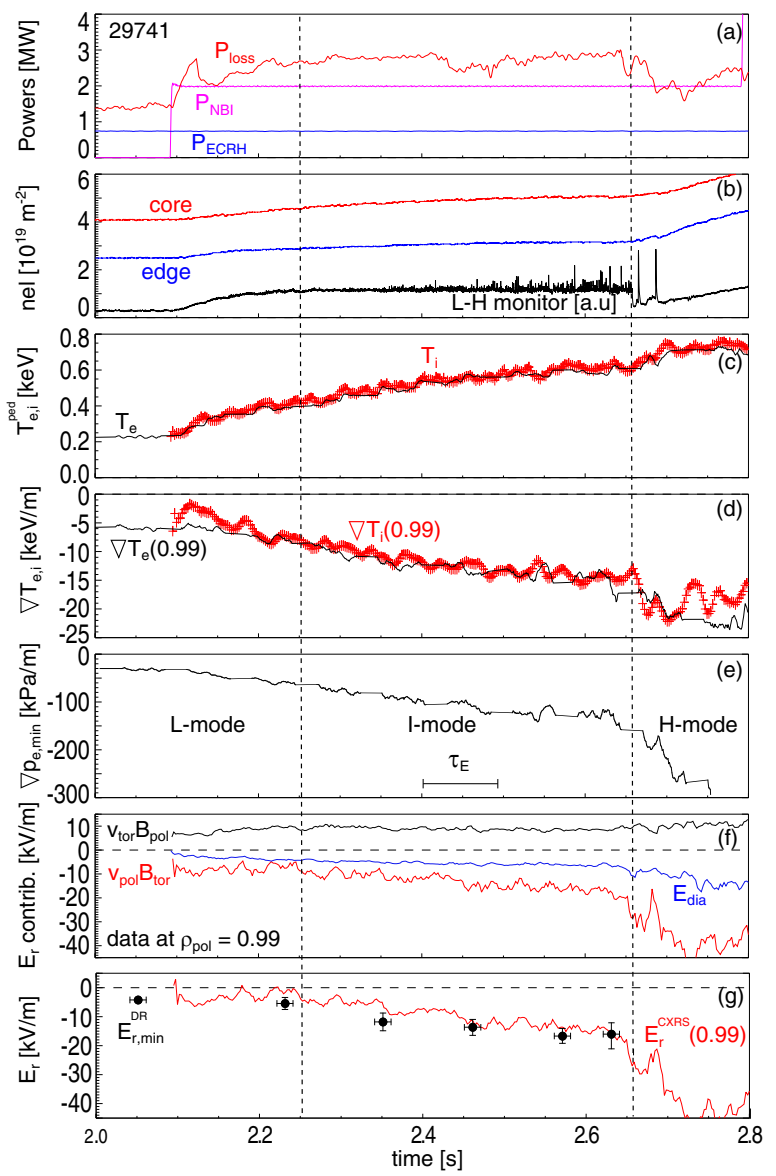

Figure 12. Time evolution of an NBI-induced I-mode in USN. Panel (a): powers; panel (b): core and edge density, as well as L-H monitor (AXUV signal in upper divertor); panel (c): pedestal top values of $T_{e}$ and $T_{i}$, taken at $\rho_{\text {pol }} \approx 0.97$; panel $(d): T_{e}$ and $T_{i}$ pedestal (steep) gradients, taken at $\rho_{\text {pol }} \approx 0.99$; panel (e): minimum of the electron pressure gradient; panel $(f)$ : the three contributions to the measurement of $E_{r}^{C X R S}$ calculated for the CXRS channel at $\rho_{\text {pol }} \approx 0.99$; panel $(g): E_{r}^{C X R S}$ at $\rho_{\text {pol }} \approx 0.99$ (from panel (f)) and $E_{r}^{D R}$ points.

As shown in panel (a), the ECRH was first turned on with $0.75 \mathrm{MW}$, the plasma remains in L-mode. Later the NBI source 3 is turned on with about $2 \mathrm{MW}$ yielding $P_{\text {loss }} \approx 2.8 \mathrm{MW}$. The L-I transition, triggered by the arrival of a sawtooth pulse at the plasma edge, takes place at about $2.25 \mathrm{~s}$ and the I-mode pedestal develops gradually until the transition to H-mode at 
$2.66 \mathrm{~s}$, both times are indicated in the figure by vertical dashed lines. The I-H transition is followed by two type-III ELMs before the EM-free phase, one of the rare occurrences of typeIII ELMs just after the I-H transition. As in the discharge discussed in the previous section, a moderate slow density increase occurs (panel b). The development of the temperature pedestal is clearly indicated by the slow increase of $T_{e}$ and $T_{i}$ at the pedestal top (panel c), and by the steepening of the edge $\nabla T_{e}$ and $\nabla T_{i}$ in panel (d). The oscillations on the signal in panels (c) and (d) are caused by the sawtooth crash pulses. As already indicated above and shown in panel (e), the electron pressure gradient also steepens and its minimum value drops from $-30 \mathrm{kPa} / \mathrm{m}$ in the L-mode with ECRH only, down to $-80 \mathrm{kPa} / \mathrm{m}$ at the L-I transition to reach $-160 \mathrm{kPa} / \mathrm{m}$ at the end of the I-mode, just before the I-H transition, it further decreases in $\mathrm{H}$ mode. In panel (f) and (g) we analyse the edge radial electric field. As mentioned in section $3, E_{r}$ can be deduced from the ion force balance, Eq. 1 applied here to $\mathrm{B}^{5+}$ measured by the edge CXRS poloidal and toroidal systems. In panel (f) we plot the time traces of the three contributions to $E_{r}$ : from poloidal and toroidal velocities, $\mathrm{v}_{\text {pol }} \mathrm{B}_{\text {tor }}$ and $\mathrm{v}_{\text {tor }} \mathrm{B}_{\text {pol }}$ respectively, as well as from the diamagnetic term, $\nabla p_{i} /\left(Z n_{i} e\right)$ with $\mathrm{Z}=5$ for boron. These quantities are taken at $\rho_{\text {pol }} \approx 0.99$ which is the position of one CXRS channel close to the position of $E_{r, \text { min }}$ as will be shown later. The ion pressure gradient is calculated using a neighbour channel at $\rho_{\text {pol }} \approx 0.98$. As already indicated for the H-mode in [25], here also the CXRS measurement of the $E_{r}$ well using this impurity is dominated by the contribution of the poloidal velocity, while the diamagnetic and $v_{t o r} B_{\text {pol }}$ contributions almost cancel each other. In panel $(\mathrm{g})$ of figure 12 we indicate the value of $E_{r}^{C X R S}$ at $\rho_{\text {pol }} \approx 0.99$ calculated with these values and the minimum of $E_{r}^{D R}$ yielded by the Doppler reflectometry. Note that $E_{r}^{D R}$ cannot be estimated after the I-H transition because the level of fluctuations is very low and results in a loss of the back-scattered signal. Due to the frequency sweep required to scan the radial position, $E_{r, m i n}^{D R}$ is only measured every $100 \mathrm{~ms}$, during each frequency scan, and the exact time indicated in the legend depends on the actual radial position of this minimum. These two measurements indicate that the $E_{r}$ well becomes more negative from $\mathrm{L}$ to $\mathrm{H}$ mode during the I-mode. The L-I transition occurs for $E_{r, \text { min }} \approx-6 \mathrm{kV} / \mathrm{m}$. During the I-mode $E_{r, \text { min }}$ becomes more negative and the I-H transition occurs for $E_{r, \text { min }} \approx-18 \mathrm{kV} / \mathrm{m}$. In the H-mode $E_{r, \min }$ becomes clearly much more negative.

The Doppler reflectometry yields the $E_{r}$ profiles shown in figure 13, where we also report for each of the $E_{r, \text { min }}^{D R}$ time points the corresponding value of $E_{r}^{C X R S}$ at $\rho_{\text {pol }} \approx 0.99$, (identical 


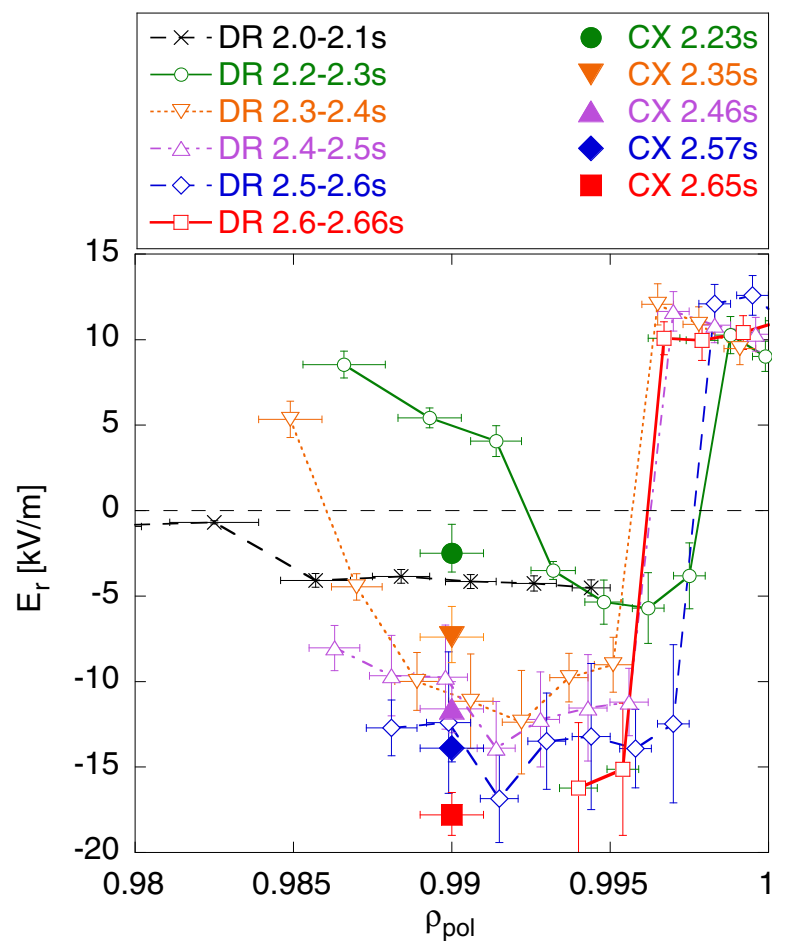

Figure 13. $E_{r}^{D R}$ profiles from the Doppler reflectometry for discharge 29741, the time intervals for each profile are indicated in the legend. The $E_{r}$ values from the CXRS channel closest to $E_{r, \min }^{D R}$ are also shown, whereby the time points, which are indicated in the legend, are chosen such that they correspond to those of $E_{r, m i n}^{D R}$ in each frequency sweep.

to that of panel $(\mathrm{g}))$. The $E_{r}^{D R}$ profile is quite flat in the L-mode with ECRH only and the minimum poorly determined. In the scan $2.2-2.3 \mathrm{~s}$, during which the L-I transition occurs, $E_{r, \text { min }}^{D R}$ is well defined, at $\rho_{p o l} \approx 0.996$. An inwards radial shift of $E_{r, \text { min }}^{D R}$ is clearly visible in the following time intervals which is then close to $\rho_{p o l}=0.99$ at the end of the I-mode, and therefore close to the CXRS measurement. This indicates that the two diagnostics yield very similar values and that the somewhat less negative value of $E_{r}^{C X R S}$ in the L-mode and at the beginning of the I-mode is due to the radial location of the channel. The relative uncertainties of the position are indicated by the error bars in figure 13. The absolute uncertainties for the two diagnostics are larger, due to those in the equilibrium and in addition to those on the density profile for the Doppler reflectometry. Due to this and because we do not have a complete profile from the CXRS diagnostics, we cannot exclude that a complete $E_{r}^{C X R S}$ profile might yield a somewhat more negative $E_{r, \text { min }}$ located at $\rho_{\text {pol }}>0.99$.

A further interesting aspect in this discharge series is related to the fluctuations observed to- 
wards the end of the I-mode, already pointed out in the previous section. The L-H monitor plotted in panel (b) of figure 12 exhibits an increase of the fluctuations, also observed in the original improved L-mode experiments, but not understood, [5]. In discharge 27943 of the present series, the Doppler reflectometry was operated at a fixed frequency chosen such that the measurement was located close to the radial position of $E_{r, m i n}^{D R}$. This setting enabled us to measure continuously the turbulence behaviour at almost the same radial position as the density variation is small. This analysis indicates that the density fluctuations exhibit an increasingly more intermittent character as the I-mode pedestal develops: there are longer phases with a low fluctuation level ending with a strong, very short ( 3 to $10 \mu$ s) burst of large fluctuations, $[37,38]$. It should be emphasised that the bursts are much too fast to be ELMs and that their occurrence is very erratic. As the pedestal increases, the time interval between the bursts is getting longer and their amplitude larger. The peaks observed in the L-H monitor and in $D_{\alpha}$ are caused by these turbulence bursts which seem to release energy and particle from the pedestal which then flow into the divertor region. The spikes measured by the L$\mathrm{H}$ monitor are longer in time than the turbulence bursts measured by the reflectometer most likely due to the time constant of the edge/divertor physics mechanisms which lead to the emitted light. The origin of these turbulence bursts may be linked to the development of the pedestal temperature gradient as discussed in detail in [37]. Very recent more detailed measurements of $E_{r}$ and turbulence are reported in [38].

In this experimental series, we also performed two exploratory shots in which we applied 3D magnetic perturbations (MPs) with toroidal mode number $n=2$, otherwise used for ELM mitigation, [39, 40]. In one of them (29746) the perturbation was "resonant", i.e. the perturbation was essentially parallel to the field lines at the plasma edge, in the other case (29745) the perturbation was "non-resonant".

The time traces of some relevant parameters are plotted in figure 14. The MPs were turned on during the low power L-mode phase, therefore long before the L-I transition. The reference discharge without MPs is 29741 , figure 12, in which the L-I transition occurred about $150 \mathrm{~ms}$ after applying the NBI power and the I-mode pedestal developed over more than $300 \mathrm{~ms}$, followed by the transition to H-mode. In the discharge with the non-resonant configuration (left panels of figure 14), the L-I transition already occurs $75 \mathrm{~ms}$ after the application of the NBI, the I-mode developed quickly over 95ms until the I-H transition 

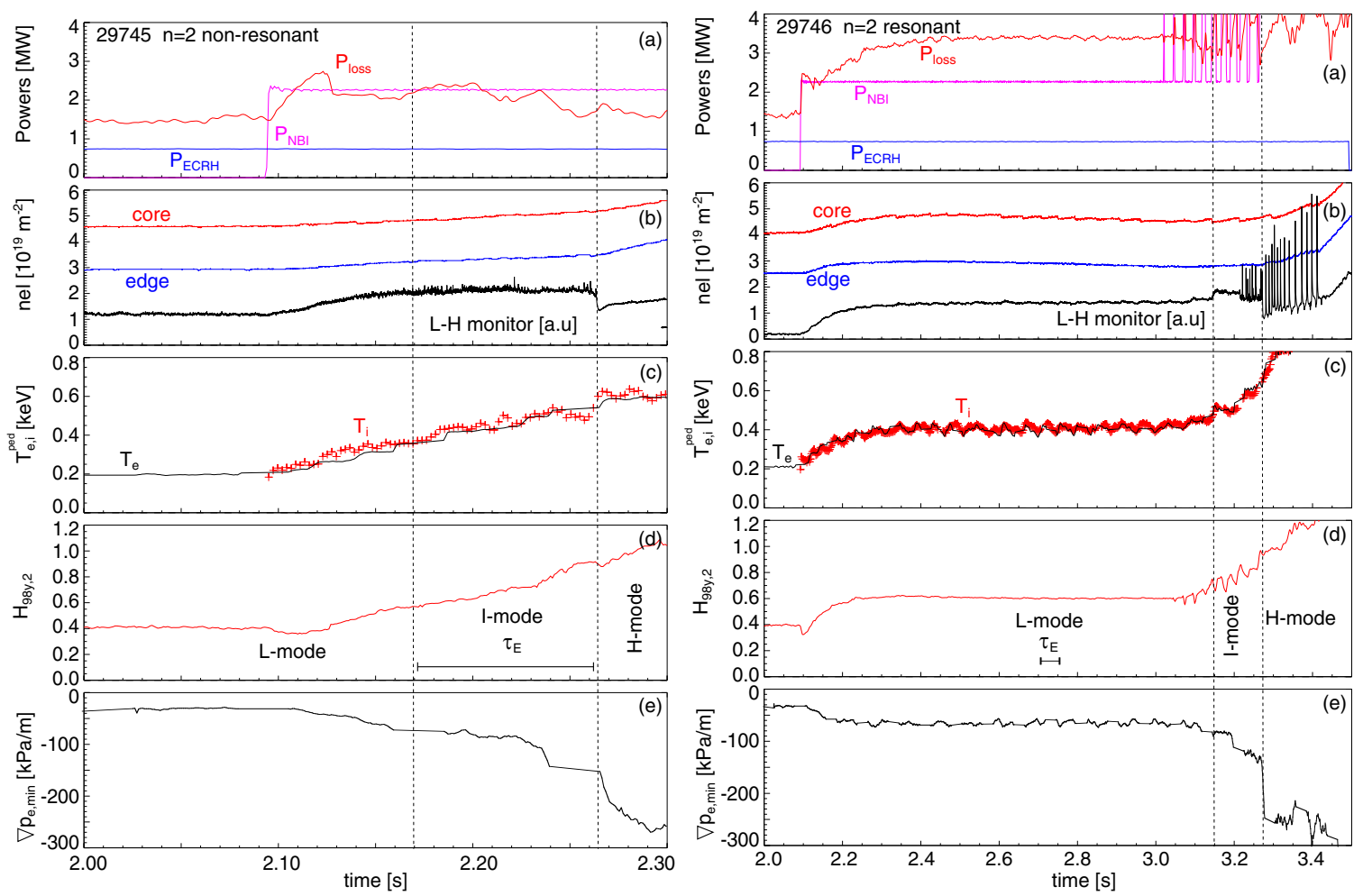

Figure 14. The two discharges with non-resonant and resonant magnetic perturbations, left and right panels respectively. Note the different time intervals. In both cases the MPS were turned on at 1.5s, reached the required current after 100ms and remained at this value until they were turned off at 3.2s which also takes $100 \mathrm{~ms}$ back to zero. Panel (a): heating powers. Panel (b): core and edge line-averageddensities. Panel (c): pedestal temperatures. Panel $(d)$ : confinement enhancement factor. Panel (e): minimum of $\nabla p_{e}$ at the plasma edge, $\rho_{\text {pol }} \approx 0.99$. The L-I and I-H transitions are indicated by the vertical dashed lines.

occurs. This is faster than in the reference discharge. At the I-H transition pedestal and confinement values are very similar to those measured at the I-H transition in the absence of MPs. Therefore, the non-resonant magnetic perturbation seems to slightly favour the occurrence and the development of the I-mode and does not hamper the occurrence the I-H transition. The respective values of $P_{l o s s}$ are about $10 \%$ lower than in the reference discharge. In contrast, the resonant $n=2$ perturbation prevents the L-I transition to occur during the first NBI power step as shown in the right panels of figure 14. Indeed, this discharge remains in a steady L-mode with $H_{98} \approx 0.6$ for one second after the NBI has been turned on, despite $P_{\text {loss }} \approx 3.4 \mathrm{MW}$, which is $0.8 \mathrm{MW}$ higher than $P_{L-I}$ in the reference discharge. The transitions to I-mode and then H-mode occur when the NBI is ramped up. The I-mode is very short 
because the power is increased, a clear transition to H-mode seems to occur at $3.27 \mathrm{~s}$, indicated in the figure, followed quickly by ELMs. The occurrence of the ELMs is possibly due to the higher heating power, whereby the MPs might also play a role. The phase before this time, starting at $3.215 \mathrm{~s}$, which also exhibits spikes on the LH monitor is not identified. It might be the I-mode, the spikes being the fluctuations mentioned above and related with the intermittent character of turbulence, or it might be a weak H-mode, but this is questionable because edge temperatures and density do not increase. Clearly, further I-mode experiments using the MPs should be performed.

\section{Properties of the L-I and I-H transitions}

In this section we present analyses of the L-I and I-H transitions, based on the discharges performed since 2009. They are similar to the examples presented in the previous section, whereas the density and to some extent the magnetic field have been varied. The magnetic configurations are either LSNrev or USN, as specified in some of the plots. We found no difference between the two configurations, as shown below. In most of the cases, the power has been varied during the discharge such that we collected data in L, I and H mode. The L-I transition is identified as described in section 2 . The I-H transition is easily and accurately identified by the abrupt drop in the divertor signals and by the strong increase of the edge density

\subsection{Overview of the L-I power threshold}

As reported for ASDEX Upgrade, [41], and Alcator C-Mod, [9], the heating power required to induce the L-I transition, $P_{L-I}$, increases with the line-averaged density. This is illustrated in figure 15 for the data of the present work.

As shown in figure 15 panel (a), $P_{L-I}$ clearly increases with the core line-averaged density, following an offset linear dependence, illustrated by the dashed line which corresponds to a least square fit. Such density dependence is also observed in Alcator CMod, [16]. The offset is strongly reduced when $P_{L-I}$ is plotted versus the edge line-averaged density, as show in panel (b) of figure 15. This is in agreement with the hypothesis that the physics mechanism of the L-I transition acts at the plasma edge. Two data points around $\bar{n}_{e} \approx 2.5 \times 10^{19} \mathrm{~m}^{-3}$, marked as "ECRH low $n_{e}$ " in these two plots, exhibit high $P_{L-I}$ values which are attributed to the dominant electron heating, as discussed in the next subsection. 

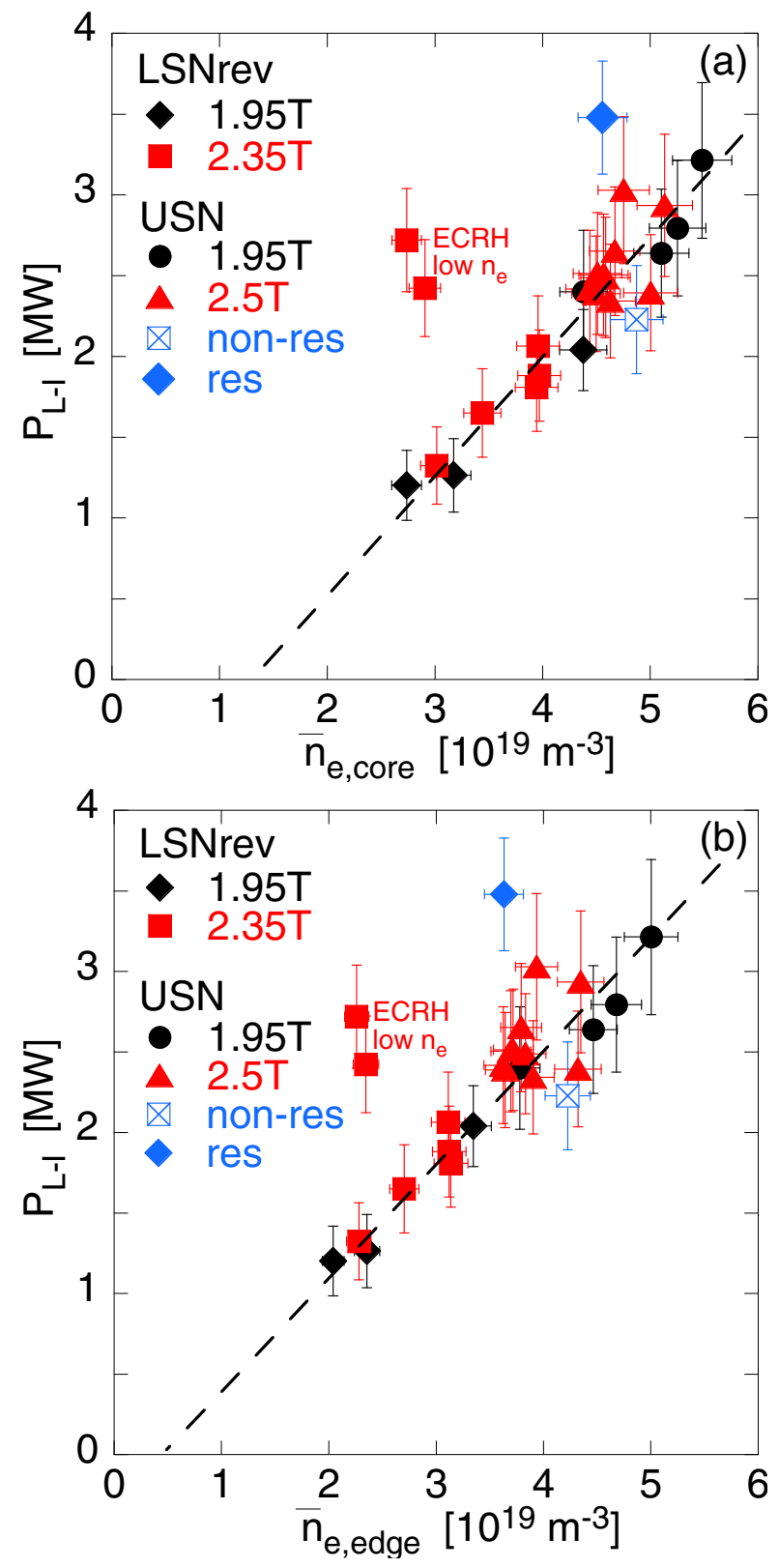

Figure 15. Density dependence of the power required to induce the L-I transition: upper plot core line-averaged density, lower plot edge line-averaged density. The different magnetic field values and configurations are indicated by different symbols. The two points with resonant and non-resonant magnetic perturbations are at $B_{T}=2.5$ $T$.

These points were not included in the fits.

In these plots we indicate three values of the magnetic field, as well as data obtained in the LSNrev and USN configurations. We remind here that for both configurations the ion $\nabla B$ drift is away from the active $\mathrm{X}$-point. The good alignment of all the points indicates that $P_{L-I}$ 
does not depend on the position of the X-point, bottom or top of the machine, as long as the ion $\nabla B$ drift is away from the active X-point. As in ASDEX Upgrade the geometry of the upper and lower divertor are very different, this suggests that the divertor conditions do not play a dominant role in the L-I transition. Figure 15 also shows that the points at different magnetic field values are rather well aligned, indicating that the magnetic field dependence of $P_{L-I}$ is weak, in the rather limited range $(1.95-2.5 \mathrm{~T})$ available here. From this limited dataset and taking the experimental uncertainties into account, we estimate the $B_{T}$ dependence to lie between $B_{T}^{0.15}$ and $B_{T}^{0.45}$. A more accurate assessment would require a wider range in $B_{T}$ values. We also note that no significant dependence of $P_{L-I}$ upon $I_{p}$ is found in our narrow range $0.8-1.0 \mathrm{MA}$. The weak $B_{T}$ dependence is in agreement with the recent findings in Alcator C-Mod carried out on a much wider range of magnetic field, [16]. This is in contrast to the L-H transition, for which, as well known, $P_{L-H}$ is proportional to $B_{T}^{0.8}$, [42]. This suggests that at least some elements of the physics mechanism of the L-I transition are different from those of the L-H transition, which is possibly linked to the difference reaction of particle transport in the two transitions.

The points from the two discharges in which the MPs have been applied, as described above, are also reported in figure 15, but not included in the fit. For the non-resonant case which somewhat favours the transition to I-mode, $P_{L-I}$ is at the lower boundary of the other values. In contrast, the resonant perturbation clearly hampers the occurrence of the I-mode, as reflected by a significant increase of $P_{L-I}$. The effect of the magnetic perturbations on the I-mode is in contrast to our results on their impact on the L-H transition in the favourable configuration, reported in [43,44]. Indeed, the application of magnetic perturbations with $\mathrm{n}=2$, as those used in the present work, was found to increase the L-H power threshold significantly only above a density of about $6 \times 10^{19} \mathrm{~m}^{-3}$, which is higher than that used here. This might also reflect differences in the transition physics. It should, however, also be underlined that $P_{L-I}$ being about two times higher than $P_{L-H}$ in the density range of these experiments, the values of the plasma parameters, e.g. rotation and collisionality, which affect the action of the magnetic perturbations on the plasma, were different for the L-H and L-I transitions.

In order to also place our results on $P_{L-I}$ in the context of the H-mode power threshold, in figure 16 we compare $P_{L-I}$ with the power at the transition from I to $\mathrm{H}$-mode, $P_{I-H}$, and with the usual L-H threshold power in the favourable configuration LSNfav, $P_{L-H}$. These points for 


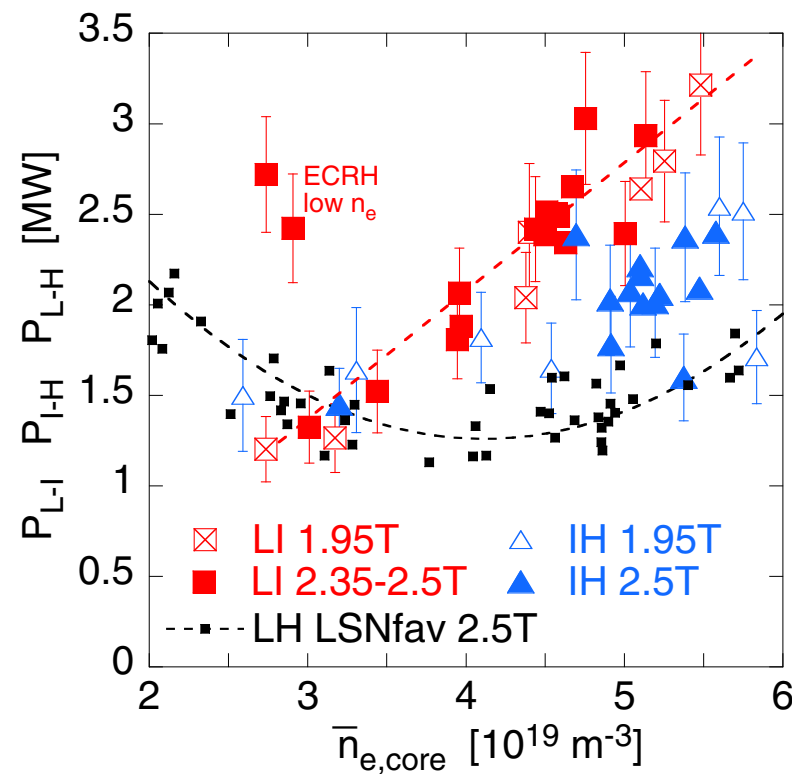

Figure 16. Density dependence of the power required for the $L-I, I-H$ and $L-H$ transitions for two magnetic field values, according to the symbols indicated in the legend. The points with MPs are not displayed.

$P_{L-H}$, taken from the set of discharges at a plasma current of $1 \mathrm{MA}$ reported in [44], exhibit the well-known non-monotonic density dependence with a minimum at $\bar{n}_{e} \approx 4 \times 10^{19} \mathrm{~m}^{-3}$, discussed in detail in $[44,45]$. This minimum separates the so-called low and high density branches of the H-mode threshold. In the high density branch $P_{L-I}$ is clearly higher than $P_{L-H}$ which reflects the well-known property that the L-H transition power is higher in the unfavourable configuration, see e.g. [46] and references therein. As indicated in the previous section, the I-mode pedestal often evolves with time at constant input power and the I-H transition can occur at a power which is below $P_{L-I}$ due to the reduction of the Ohmic power and to the $\mathrm{dW} / \mathrm{dt}$ correction. This is clearly shown by the $P_{I-H}$ points in the high density branch, for both magnetic field values. The scatter in $P_{I-H}$ is large because it depends on the rapidity of the time evolution of the I-mode which is linked with the heating power itself. If the applied power is significantly above $P_{L-I}$, the I-mode evolves quickly and $P_{I-H}$ is rather high, whereas if the heating power is just above $P_{L-I}$, the time evolution of the I-mode is slow, the pedestal has time to develop and the conditions for the I-H transition, e.g. $E_{r}$ well deep enough and step pressure gradient are reached at lower power. In contrast, towards low density, $P_{L-I}$ crosses the low density branch of $P_{L-H}$ for NBI while it is higher with ECRH, as indicated by two points labelled "ECRH low $n_{e}$ ". This suggests that, at sufficiently low 
density, it might be possible to access the I-mode with about the same installed heating power as required for the $\mathrm{H}$-mode, but it remains to demonstrate experimentally that the density can be then increased keeping the I-mode with an adequate increase of the heating power but without triggering the transition to $\mathrm{H}$-mode.

\subsection{Heating method and edge ion heat flux at the L-I transition}

The increase of $P_{L-H}$ towards low density has been interpreted in ASDEX Upgrade by the fact that the neoclassical edge radial electric field well plays a key role in the L-H transition physics, [35, 47]. As the $E_{r}$ well is mainly driven by the main ions pressure gradient, $[33,48]$, the ion heat flux at the plasma edge, $Q_{i, e d g e}$, is expected to play a key role, as indeed demonstrated experimentally, [45, 49]. It has been shown that $Q_{i, e d g e}$ at the L-H transition increases linearly with density, $Q_{i, e d g e}^{L-H, f i t}=a_{L H} \bar{n}_{e}$, in MW and $10^{19} \mathrm{~m}^{-3}$, the coefficient $a_{L H}$ being equal to 0.18 and 0.45 in deuterium and hydrogen respectively. Thus the increase of $P_{L-H}$ as density is reduced in the low density branch is attributed to the fact that more heating power is required to reach the value of $Q_{i, e d g e}$ necessary for the $\mathrm{L}-\mathrm{H}$ transition because the collisional electron-ion energy exchange diminishes towards low density. This effect is particularly strong when ECRH is used.

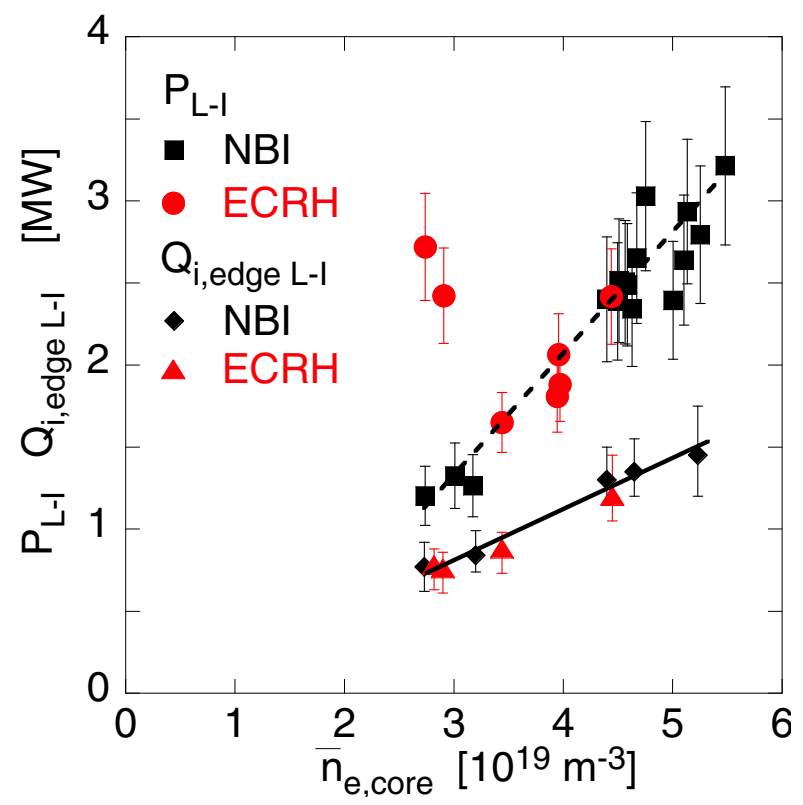

Figure 17. $P_{L-I}$ and $Q_{i, e d g e}$ versus density for L-I transitions with NBI and ECRH.

Following the same argumentation, one may tentatively explain the two high values of 
$P_{L-I}$ with ECRH at low density by the fact the L-I transition also requires a minimum edge ion heat flux. The results are shown in figure 17 which displays $P_{L-I}$ and $Q_{i, e d g e}$ at the L-I transition versus density for cases with NBI and ECRH. We find no difference in $P_{L-I}$ between ECRH and NBI for densities above $\approx 3.3 \times 10^{19} \mathrm{~m}^{-3}$, which is in line with the fact that the ion heat fluxes at the plasma edge are every similar for both heating methods, as shown in figure 8. In contrast, below $3.3 \times 10^{19} \mathrm{~m}^{-3} P_{L-I}$ is very high for ECRH, but aligned with the other points when NBI is used. The power balance analysis allows to separate the electron and ion channels up to the edge if the coupling is not too high, $\bar{n}_{e}<5.5 \times 10^{19} \mathrm{~m}^{-3}$ in the present cases. The $Q_{i, e d g e}$ values at the L-I transition plotted in figure 17 versus density are well aligned, including those corresponding to the two high $P_{L-I}$ values with ECRH. A linear fit yields $Q_{i, e d g e}^{L-I, f i t}=0.3 \bar{n}_{e}$ in MW and $10^{19} \mathrm{~m}^{-3}$. In contrast to $P_{L-I}$, the density dependence of $Q_{i, e d g e}$ has a negligible off-set. This is qualitatively very similar to the results found for the L-H transition, whereby the slope value of 0.3 is higher than $a_{L H}$ for the L-H transition in deuterium, which is in agreement with $P_{L-I}>P_{L-H}$, but lower than $a_{L H}$ in hydrogen for which $P_{L-H}$ is indeed higher than $P_{L-I}$ in deuterium, see [49] for comparison.

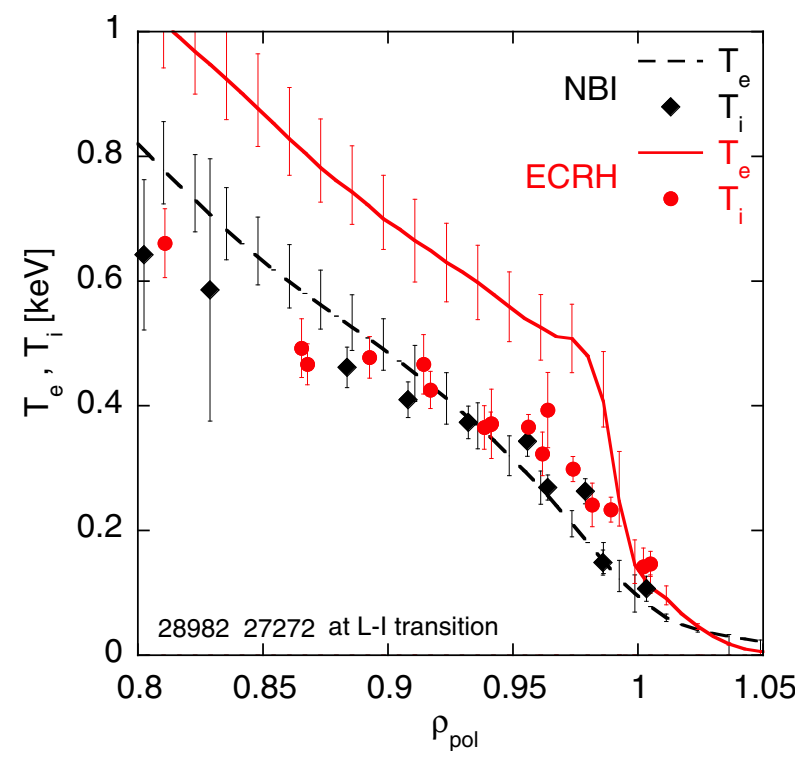

Figure 18. Electron and ion temperature profiles at the L-I transition induced with NBI or ECRH for the lowest density points of figure 17

This is confirmed by the electron and ion edge profiles plotted in figure 18 at the L-I transition for the lowest density with NBI and ECRH. For the NBI case $T_{i} \approx T_{e}$, but with ECRH only the $T_{i}$ profile is very close to that with NBI, while $T_{e}$ is much higher and the edge 
gradient steeper. This is similar to what we observed below the usual L-H threshold in the low density branch with ECRH, [35].

It should be underlined that, as shown by figure 16, the increase of the threshold power with ECRH in the low density branch is stronger for the L-I transition than for the usual L-H transition. This can be explained as follows: $Q_{i, \text { edge }}^{L-I}$ being higher than $Q_{i, e d g e}^{L-H}$ more ECRH power is required for the L-I transition than for the L-H transition. As the electron-ion collisional coupling, $p_{e, i} \propto n_{e} n_{i}\left(T_{e}-T_{i}\right) T_{e}^{-3 / 2}$, saturates with increasing $T_{e}$, as illustrated with experimental data in $[45,49]$, the ECRH power necessary to establish the value of $Q_{i, e d g e}$ for the L-I transition increases strongly non-linearly compared to the ratio $Q_{i, e d g e}^{L-I} / Q_{i, e d g e}^{L-H}$. It should be underlined that in Alcator C-Mod, where the L-I transition is achieved with ICRH which is an efficient electron heating, $P_{L-I}$ also seems to increase strongly towards low density, [16] figure 15.

\subsection{Edge data at the L-I transition}

The actual physics mechanism causing the L-I transition is not identified yet. As mentioned above, the L-I transition is correlated with the appearance of the WCM and GAM, both located at the very edge of the plasma, close to the minimum of the pressure gradient or, almost equivalently of the radial electric field, $[13,14]$. It is not clear whether the heat transport reduction which induces the formation of the temperature pedestal is due to one of these two instabilities, or to their non-linear interaction, or rather to the perpendicular flow shear similarly to the L-H transition. With the aim of providing some experimental elements on this question, we investigated the electron data yielded by the IDA analysis.

We analyse the minimum of the pressure gradient, $\nabla p_{e, \min }$, at the L-I transition, plotted in figure 19 versus heating power with different symbols for ECRH and NBI. As indicated in section 3 the radial location of $\nabla p_{e, \min }$ is at the very edge, in the range $\rho_{\text {pol }} \approx 0.98-0.99$. The NBI cases suggest a decrease with $P_{\text {loss }}$ and it should be underlined that the two points for which the magnetic perturbations were applied are well aligned with the others. This shows that the magnetic perturbations influence the L-I transition by their impact on the pressure gradient. In contrast, the ECRH points exhibit a strong scatter and no trend.

In figure 20 we plot the same data as a function of density. There also, the NBI points are rather well aligned and $\nabla p_{e, \min }$ becomes more negative as density increases. In contrast to the previous figure, the ECRH points exhibit a somewhat clearer pattern whereby the most 


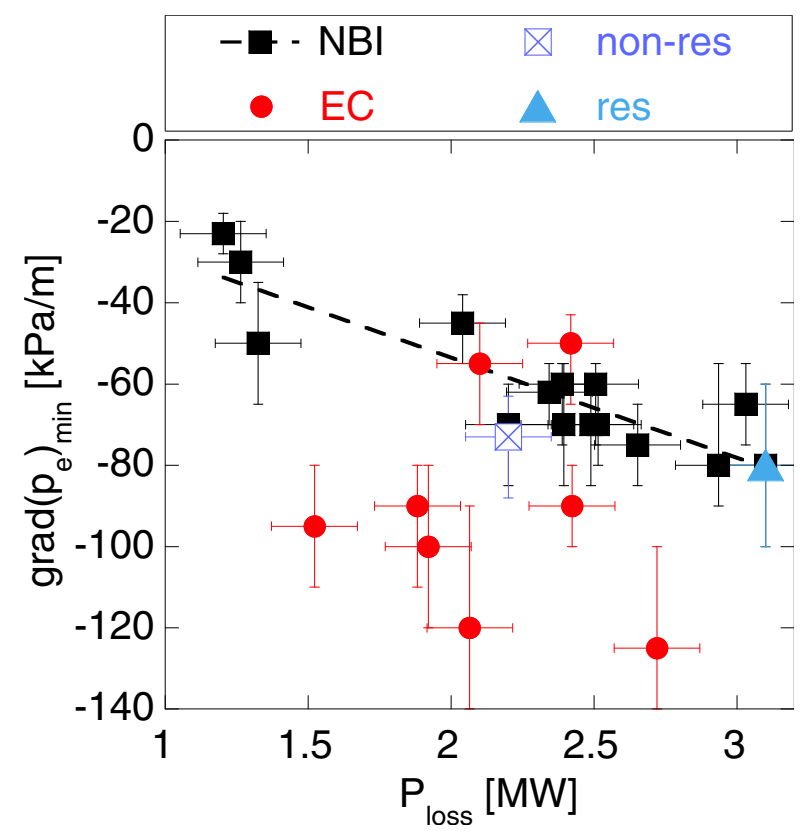

Figure 19. $\nabla p_{e, \min }$ versus $P_{\text {loss }}$ at the L-I transition for the cases indicated in the legend.

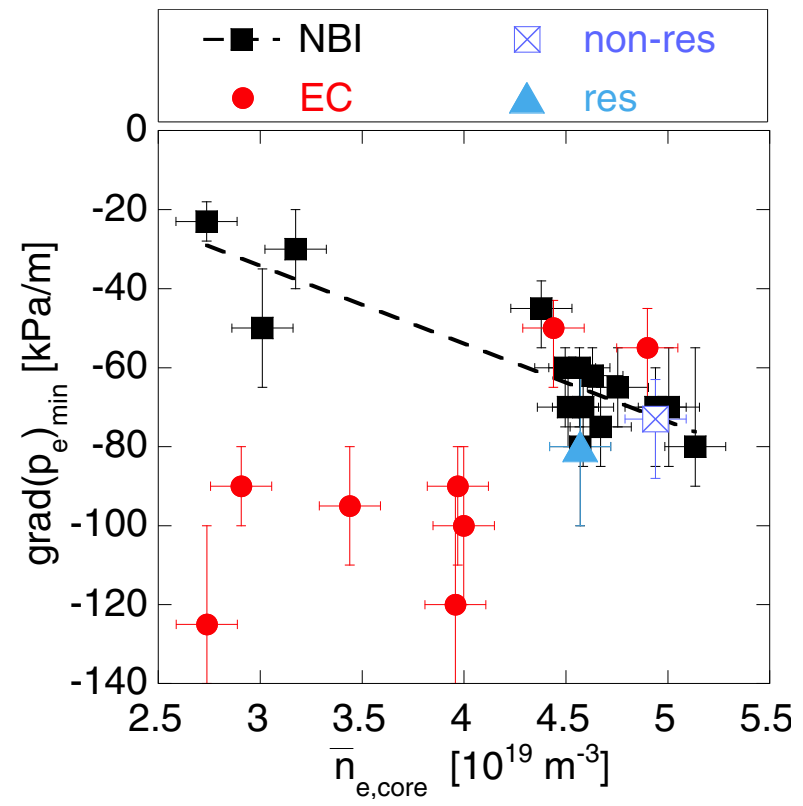

Figure 20. $\nabla p_{e, \text { min }}$ versus density at the L-I transition for the cases indicated in the legend.

negative values are found towards low densities. This is in agreement with the decoupling of the electron and ion channels at low density with ECRH and the fact that a $T_{e}$ pedestal develops before the L-I transition which yields a high pressure gradient reflected by negative values of $\nabla p_{e, \min }$ at the L-I transition. Together with our results on the edge ion heat, figure 
17, the analysis of $\nabla p_{e, \min }$ and the differences between NBI and ECRH induced L-I transitions at low density strongly suggest that the ion channel plays a key role in the physics mechanism, of the L-I transition.

We have so far very few profiles of $E_{r}$ for the L-I transition but they all yield a value for $E_{r, \text { min }}$ between -6 and $-10 \mathrm{kV} / \mathrm{m}$. With this dataset it is not possible to conclude on the dependence of $E_{r, \min }$ at the L-I transition on density or magnetic field. The values of $E_{r, \min }$ at the I$\mathrm{H}$ transition are more negative, in the range -18 to $-24 \mathrm{kV} / \mathrm{m}$. For ASDEX Upgrade it has been shown in [35] that $E_{r, \text { min }}$ is close to $-15 \mathrm{kV} / \mathrm{m}$ for the usual L-H transition at the same magnetic field value of $2.5 \mathrm{~T}$. Our present results suggest that $E_{r, \min }$ at the L-I transition might be somewhat less negative than at the usual L-H transition and somewhat more negative at the I-H transition, newer more detailed measurements are reported in [38]. Whatever the reasons for this difference are, this leaves room for other turbulence reduction mechanisms than $\nabla E_{r}$. As pointed out in [50], one possibility is the magnetic shear which is influenced by the bootstrap current at the plasma edge. The latter is indeed linked with the pressure gradient which would be in agreement with our analysis which shows the importance of the pressure gradient in the L-I transition and explains the fact that $P_{L-I}$ depends weakly on the magnetic field. As the turbulence stabilisation by $\nabla E_{r}$ and magnetic shear affect different turbulence size scales, this might be consistent with the strong reduction of particle transport at the I-H transition when $\nabla E_{r}$ is steep enough. As both bootstrap current and $E_{r}$ well depend on the pressure gradient, disentangling the two effects requires dedicated experiments with accurate measurements of all the required quantities in magnetic field scans in the favourable and unfavourable magnetic configurations. This could be the subject of further dedicated investigations. It should be noted that during the I-mode, from the L-I to I-H transitions, $\beta_{p o l}$ increases from 0.33 to 0.5 while the internal inductance, $l i$, decreases from 1.42 to 1.33 due to the broadening of the current profile. The Shafranov shift is only weakly increased, by less than $1 \mathrm{~cm}$, and the triangularity at the plasma edge by about $5 \%$. Therefore the changes in equilibrium are small and most probably not a main contributor to the observed changes in the turbulence properties. It should also be mentioned that the maximum value of $\beta_{N}$ in our I-modes was 1.2 which is rather low, such that the turbulence is expected to be mainly electrostatic. 


\section{I-mode confinement properties}

In this section we present the confinement properties of the I-mode, as well as those of the $\mathrm{L}$ and $\mathrm{H}$ modes for the corresponding discharges. We characterise the confinement by the widely used $H_{98}$ enhancement factor. In these discharges, the H-mode which develops after the I-mode is often ELM-free, which means that density and radiation losses increase strongly, leading to a loss of the H-mode after about $300 \mathrm{~ms}$ if the heating power is not increased to produce ELMs. The H-mode data presented below are taken $100 \mathrm{~ms}$ after the I-H transition, where the radiation losses are still moderate, and they generally exhibit a rather high value of $H_{98}$. Therefore, they are not necessarily representative of all the H-modes in ASDEX Upgrade, but serve as comparison to the I-mode data. We find no dependence of confinement on magnetic field in the range of our dataset. The most interesting dependences are illustrated in the following.

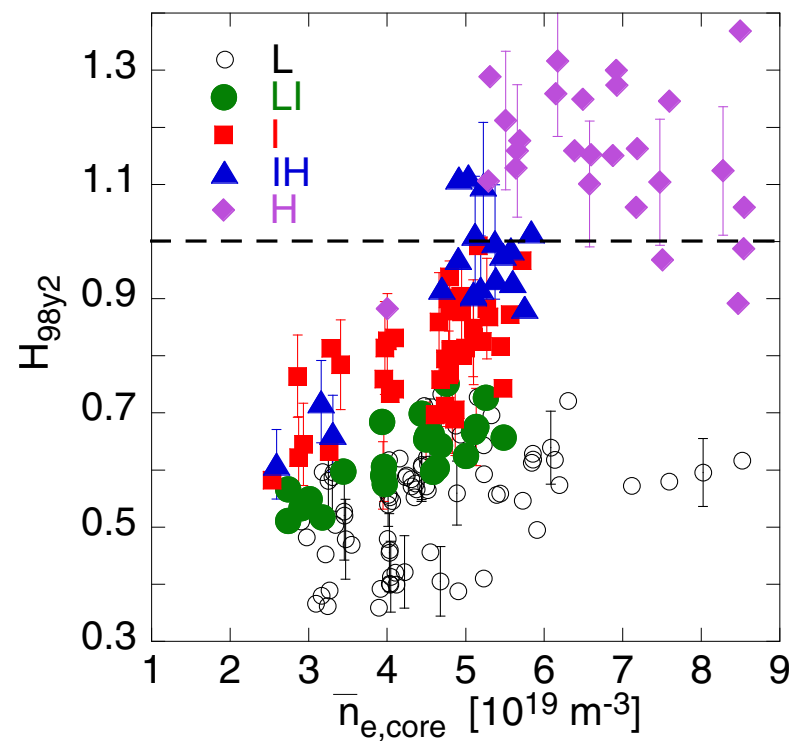

Figure 21. Density dependence of the normalised confinement, $H_{98}$ for the different plasma regimes indicated in the legend.

We start with the dependence of $H_{98}$ upon density illustrated in figure 21 which shows well the confinement range for each regime and indicates that the separation between regimes is clear. As expected, the L-mode points exhibit the lowest confinement and H-modes the highest, while the I-mode confinement lies in-between. The $H_{98}$ values at the L-I transition increase somewhat with density and are in the range $0.5-0.7$. These points build the upper boundary of the L-mode data which are indeed all below. The I-mode covers a range from the 
L-I values up to $H_{98} \approx 1$ at the I-H transition. The I-mode range exhibits overall an increase of confinement with density, despite the fact that $H_{98}$ includes a $\bar{n}_{e}^{-0.41}$ density dependence which reduces this trend. Further, it seems that the range of $H_{98}$ of the I-mode, between the LI and I-H transitions, widens towards higher values as density increases. We verified that this is not due to the range in magnetic field. The H-mode points suggest a decrease of $H_{98}$ with density which is often observed and attributed to the $\bar{n}_{e}^{-0.41}$ dependence of $H_{98}$. The density range of the I-mode data points in this figure corresponds roughly to a range in Greenwald factor between 0.2 and 0.5 . So far we did not try to increase the density in the I-mode and this upper value can certainly be pushed to higher values with gas puffing.

It is very instructive to investigate the dependence of confinement versus loss power, figure 22 which shows $H_{98}$ versus $P_{\text {loss }}$. The confinement at the L-I transition increases with heating power which is probably correlated with the density as $P_{L-I}$ increases with density. The power dependence shown in figure 22 exhibits a turn around for $H_{98} \approx 0.7$ and $P_{\text {loss }} \approx 3.2 \mathrm{MW}$. The I-mode points with higher confinement are roughly correlated with a decrease of $P_{\text {loss }}$. This is due to the spontaneous evolution of the I-mode discussed above and underlined in the plot by the $\bar{n}_{e}-P_{\text {loss }}$ trajectory for the NBI-heated discharge 26905 which is overlaid on the data points. The $\mathrm{L}, \mathrm{I}$ and $\mathrm{H}$ phases are indicated. This behaviour shows that the spontaneous evolution of the I-mode until the H-mode transition, at fixed auxiliary power, occurs for $H_{98}$ above 0.7-0.8. As the L-I transition occurs at higher values of $H_{98}$ and power for higher density, the spontaneous evolution of the I-mode might be unavoidable above a certain density in ASDEX Upgrade. However, the actual physics reason for the spontaneous evolution is not identified yet. As suggested by the NBI/ECRH comparison in figure 6, the spontaneous evolution also occurs in ECRH-heated cases. However, a possible role of the NBI blips which induce an increase of the edge pressure at each pulse cannot be excluded. Similarly, the sawtooth pulses, which are strong with NBI, could also play a role, see figure 6 . The possible impact of the toroidal rotation induced by NBI is less probable as it is known to increase the L-H threshold, [51, 45]. The uncontrolled evolution is always correlated with an increase of the density, whereby we do not know yet what is cause and consequence. Obviously further investigations are necessary to better understand and control this phenomenon which must be avoided in a future fusion reactor.

To investigate this impact of this behaviour on the confinement properties in more details, we consider two subsets of the database built as follows. We define as "steady" the I-modes 


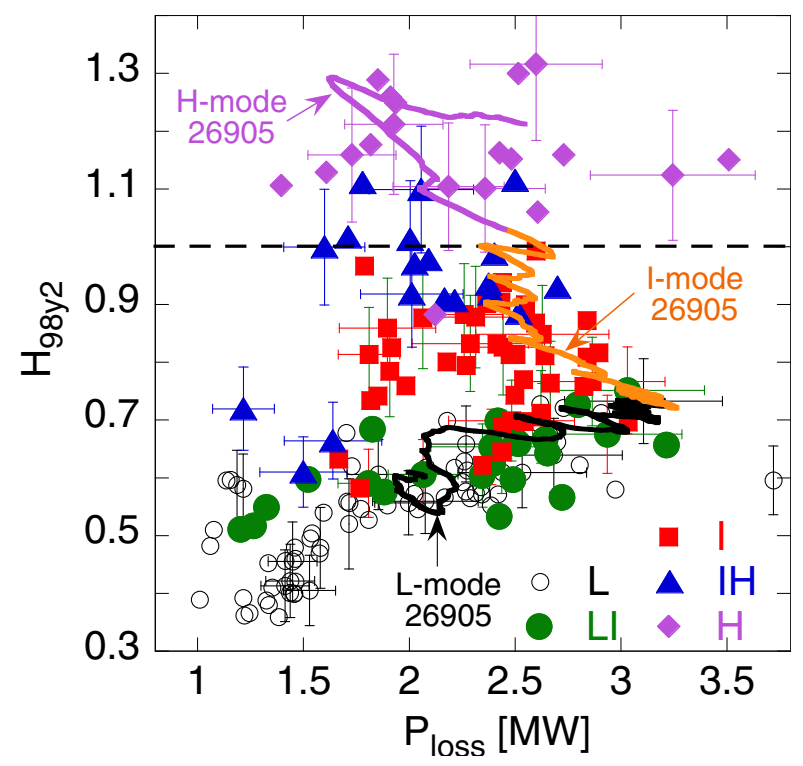

Figure 22. Power dependence of the normalised confinement, $\mathrm{H}_{98}$ for the different plasma regimes indicated in the legend.

which reach steady state after the L-I transition at fixed input heating power and $P_{\text {loss }}$. Defined as "non-steady" I-modes are those which evolve until the transition to H-mode despite fixed input power, as for instance those illustrated in figures 6 and 12. In these cases, pedestal and confinement increase in spite of the fact that the input power is kept constant and while $P_{\text {loss }}$ decreases. The L-modes are always steady at fixed heating power. I-modes in which variations of heating power do not allow us to define them as steady or not are not included.

The confinement properties of the three classes, for $I_{p}=1 \mathrm{MA}$ only, are summarised in figure 23, in panel (a) for the global confinement time, $\tau_{E}$, and in panel (b) for the $H_{98}$ factor. The plots indicate that the power dependence of $\tau_{E}$ and $H_{98}$ depends on whether the I-modes are steady or not. The L-modes exhibit a usual power degradation with $\tau_{E} \propto P_{\text {loss }}^{-0.63}$ which is in agreement with the L-mode confinement scaling [52]. For the steady I-modes the confinement time degrades as $\tau_{E} \propto P_{\text {loss }}^{-0.43}$ which is weaker than that predicted by the $\mathrm{L}$ and $\mathrm{H}$ confinement scalings. This is in agreement with the previous ASDEX Upgrade results on the Improved L-mode, [2], and with those from Alcator C-Mod, [53]. In contrast, with $\tau_{E} \propto P_{\text {loss }}^{-1.3}$ the power degradation of the non-steady I-modes is much stronger than any confinement scaling. This is due to the fact already mentioned above that in these discharges the pedestal evolves spontaneously leading to a higher plasma energy while $P_{\text {loss }}$ decreases. In the plots of figure 23 the time evolution of the non-steady I-modes goes from the right to the left and in the 

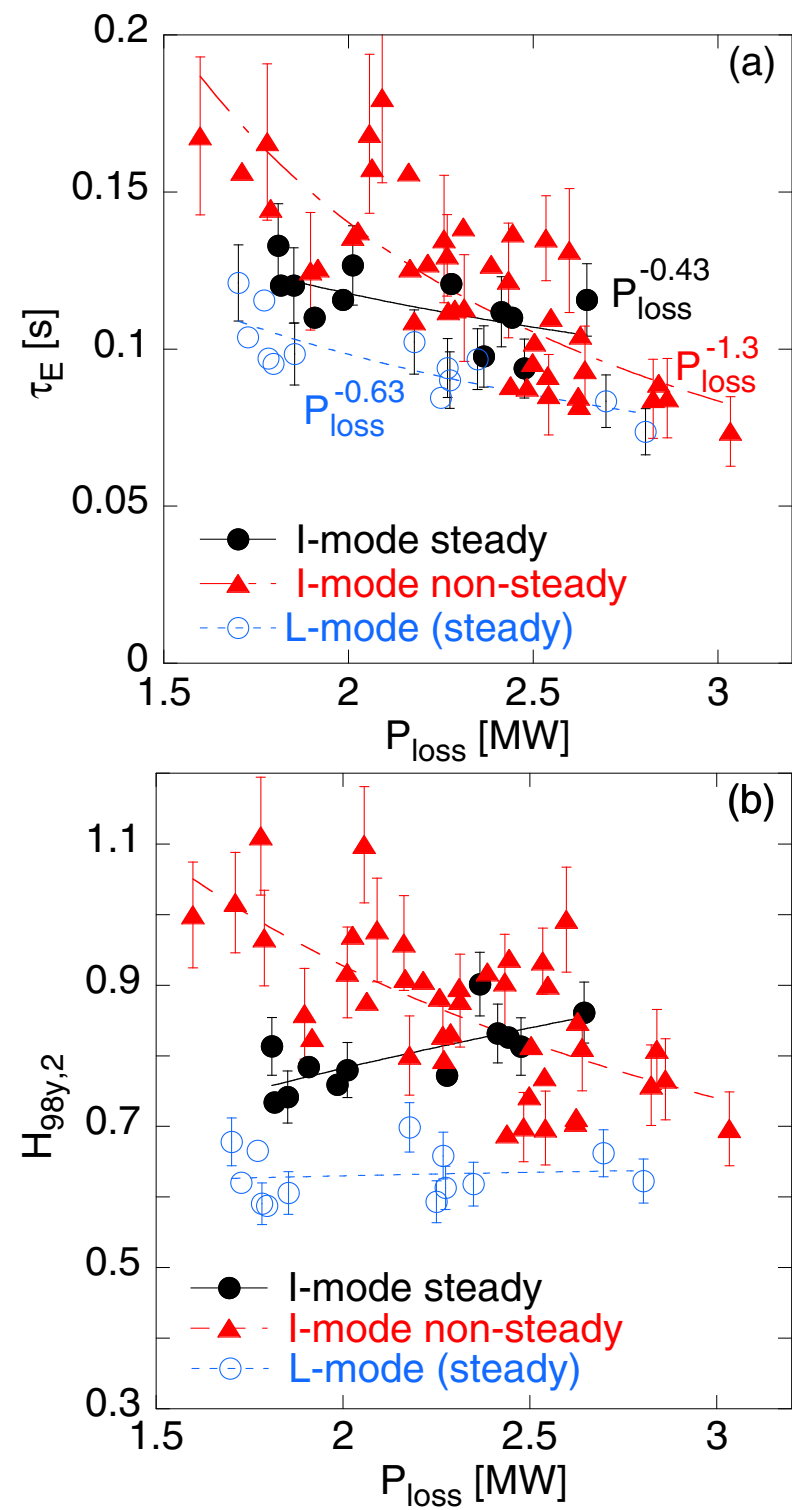

Figure 23. Confinement power degradation of the steady and non-steady I-modes, as well as L-modes. All points are from discharges at 1MA and 2.5T. Panel a: global energy confinement time. The lines are power law fits to the respective data sets and the power dependence is indicated. Panel $b: H_{98}$ for the same data points. The lines are fit to the data.

upper left corner $\tau_{E}=W / P_{\text {loss }}$ is high because the plasma energy increases thanks to the higher pedestal pressure while simultaneously $P_{l o s s}$ decreases. This strong power degradation should not be interpreted in the usual way because the confinement time is not directly and solely linked to the heating power, but rather induced by the self-amplifying development of the pedestal. In addition, the high confinement points at low power cannot be reached 
directly with the corresponding value of $P_{l o s s}$ because the latter is below the L-I transition threshold. The large scatter in both $\tau_{E}$ and $H_{98}$ for the non-steady points is due to the fact that the confinement time power degradation is not solely determined by $P_{l o s s}$.

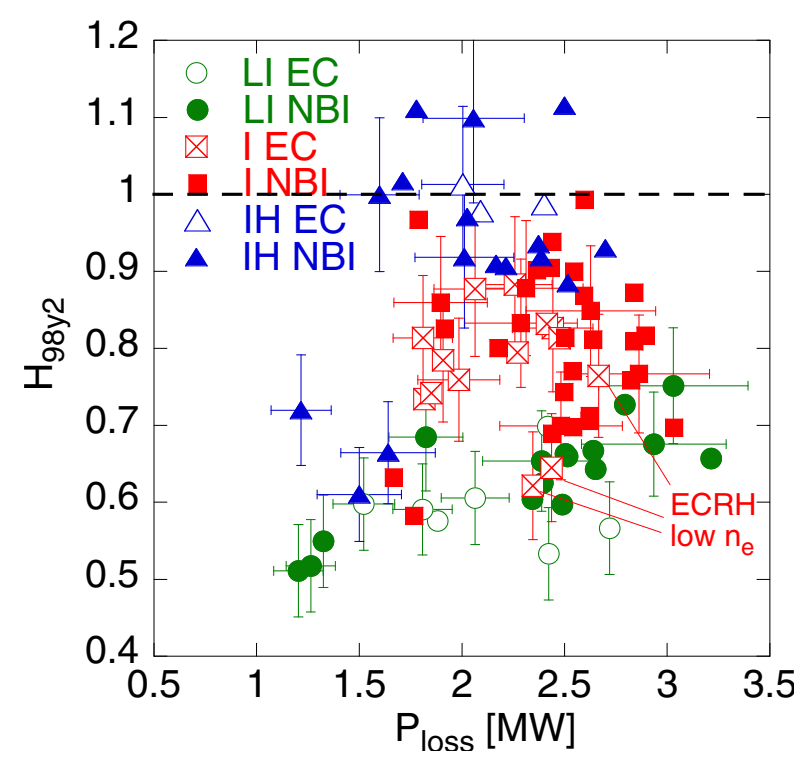

Figure 24. Power dependence of the I-mode confinement, $H_{98}$, with NBI or ECRH for the confinement phases indicated by the symbols.

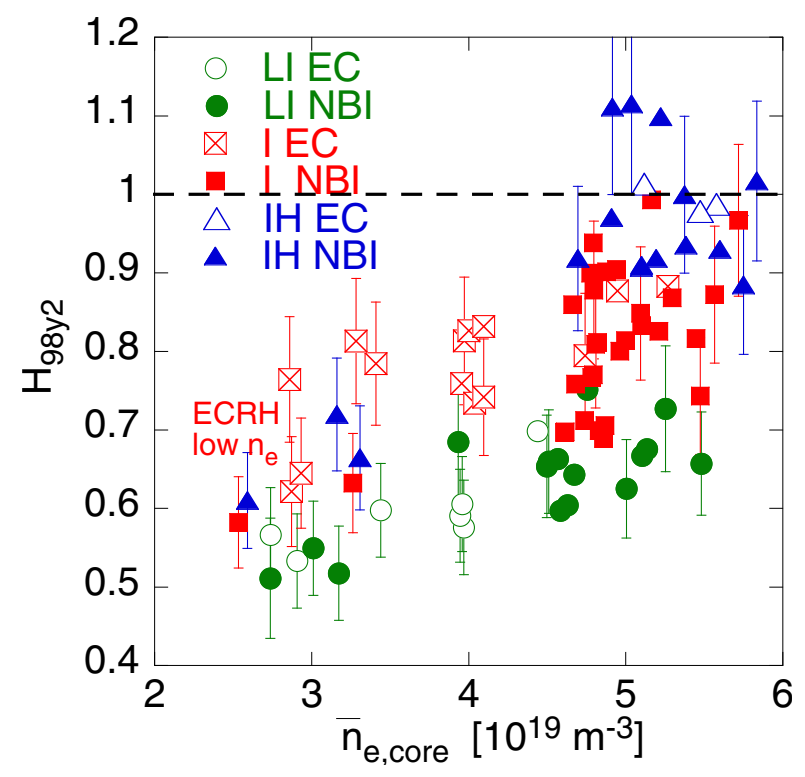

Figure 25. Density dependence of the I-mode, $\mathrm{H}_{98}$, with NBI or ECRH for the confinement phases indicated by the symbols.

As we used NBI or ECRH to obtain the I-mode, it is also instructive to investigate the 
possible impact of the heating method on the confinement properties. This is illustrated in the figures 24 and 25 again plotting $H_{98}$ versus $P_{l o s s}$ and $\bar{n}_{e}$ for the L-I, I and I-H points. These two plots indicate that confinement in the I-mode does not depend significantly on the heating method, except for the three points at very low density and with ECRH, labelled "ECRH low $n_{e}$ ". Note in particular in figure 24 that they are at high heating power but have poor confinement: In such cases the high electron contribution to the total plasma energy cannot compensate the low ion fraction. Apart from these cases and as discussed in section 3 , the heat fluxes at the pedestal do not depend on the heating method which yields very similar pedestal value for a given heating power and density. In addition, deeper in the plasma core the higher electron temperature with ECRH can compensate for the lower ion temperature, such that the plasma energy is roughly independent of the heating method under the conditions of our experiments at $\bar{n}_{e}>3.0 \times 10^{19} \mathrm{~m}^{-3}$.

The behaviour of the pedestal is a crucial element in the I-mode. We use here the electron data at the pedestal top whereby an overview is provided by plotting $T_{e}^{\text {ped }}$ versus $n_{e}^{\text {ped }}$ in figure 26. The values of $T_{e}^{\text {ped }}$ during the I-mode reach about $800 \mathrm{eV}$ at low density which is about a factor of two higher than the values at the L-I transition: this quantifies the pedestal development induced by the I-mode. At low density the maximum value of $T_{e}^{p e d}$ was not limited by the I-H transition. The highest $T_{e}^{p e d}$ values at low density $\left(n_{e}^{p e d}<2.5 \times 10^{19} \mathrm{~m}^{-3}\right)$ correspond to ECRH-heated I-modes. At somewhat higher density $\left(n_{e}^{\text {ped }}>3 \times 10^{19} \mathrm{~m}^{-3}\right)$ the enhancement of the pedestal in the I-mode is limited by the I-H transition and the excursion in $T_{e}^{p e d}$ is somewhat smaller than at lower density. The H-mode points exhibit higher $n_{e, p e d}$ values due to the density increase after the $\mathrm{I}-\mathrm{H}$ transition and the increase of $T_{e}^{\text {ped }}$ is moderate as already pointed out above.

The pedestal pressure is an important quantity in confinement studies. We document its behaviour with heating power, using here the electron pressure at the pedestal top, $p_{e}^{p e d}$, as shown in figure 27. The pedestal top pressure increases somewhat with heating power in L-mode and at the L-I transition. The increase during the I-mode, even for decreasing $P_{l o s s}$ as discussed for $H_{98}$, appears clearly: the highest values of $p_{e, p e d}$ do not correspond to the highest heating powers. As expected, the highest values of $p_{e, p e d}$ are reached in the H-mode where $p_{e}^{\text {ped }}$ seems to saturate and be rather independent of heating power.

It is well-known that the global confinement time is generally correlated with the pedestal top pressure, see $[54,55,56]$ for ASDEX Upgrade. The data plotted in figure 28 indicate 


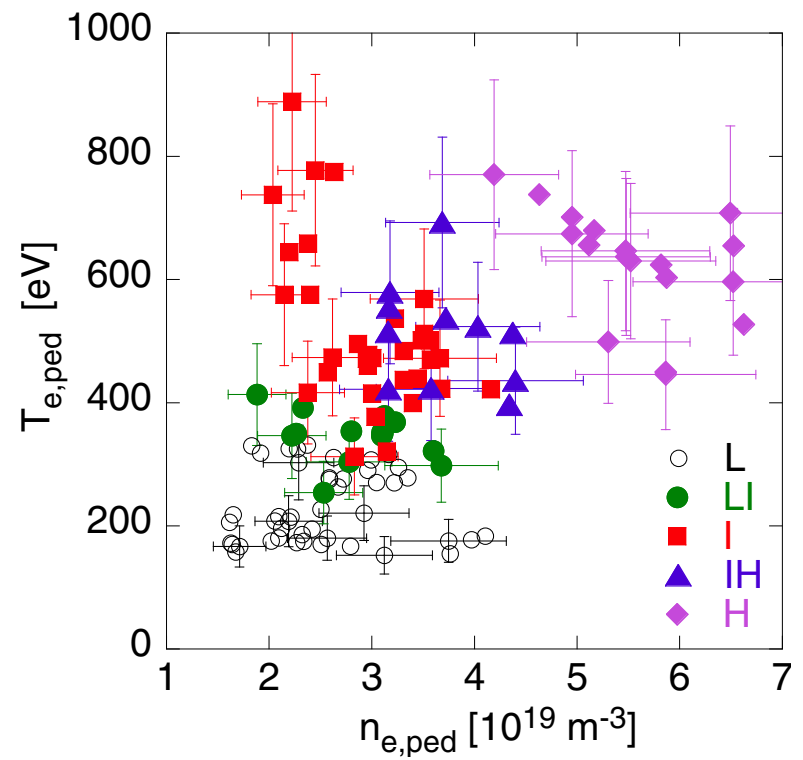

Figure 26. Pedestal top values for the different regimes: electron temperature versus density.

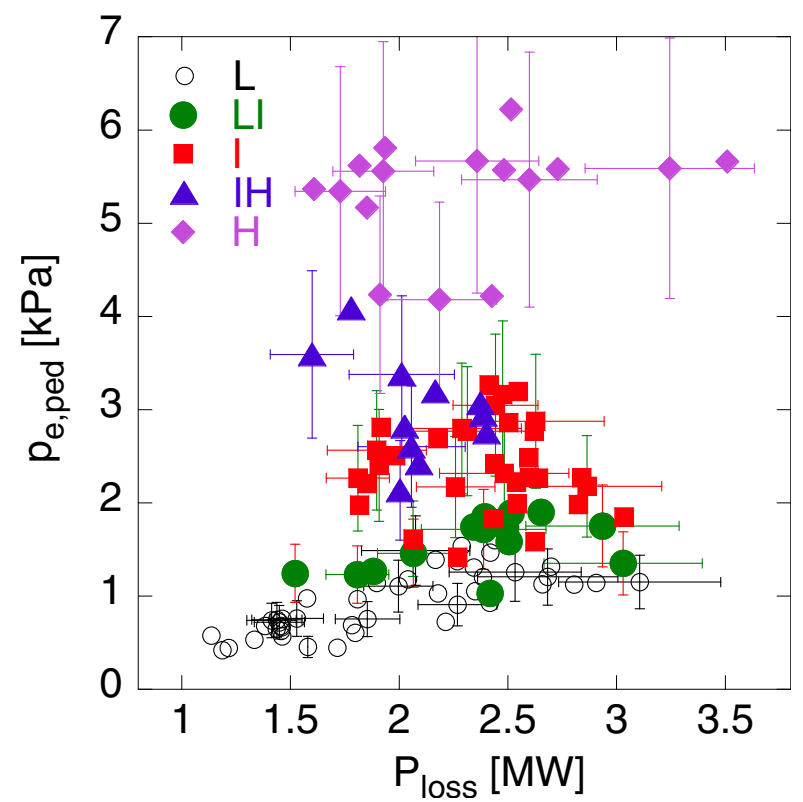

Figure 27. Pedestal electron pressure versus $P_{\text {loss }}$ for the different confinement regimes indicated by the symbols.

indeed a strong correlation between $H_{98}$ and $p_{e}^{p e d}$ for the L-mode and I-mode points up to the I-H transition. The correlation is particularly clear for the I-mode (including L-I and $\mathrm{I}-\mathrm{H}$ ) which extends over the widest range in $\mathrm{H}_{98}$. This confirms quantitatively that the improvement of the confinement time in the I-mode is mainly determined by the enhancement 
of the pedestal. The low density I-modes with ECRH exhibit high values of $p_{e}^{\text {ped }}$ but low confinement in comparison to the other points, in agreement with the explanation given above that the ion contribution is small. In contrast to the increase of $H_{98}$ with $p_{e, p e d}$ in I-mode, a saturation occurs for the H-mode points, in agreement with the fact that $H_{98}$ better describes the H-mode confinement: the pedestal is directly linked to the heating power.

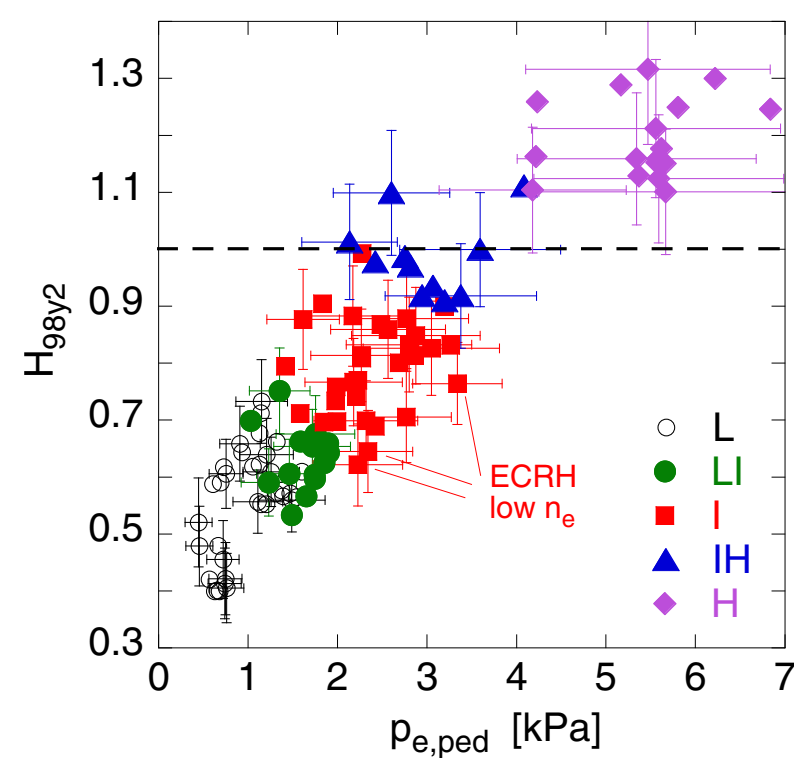

Figure 28. Correlation between $\mathrm{H}_{98}$ and pedestal top pressure for the different confinement regimes indicated by the symbols.

\section{Summary and conclusions}

The I-mode is achieved above a certain threshold in heating power if the H-mode is avoided by choosing conditions in which the ion $\nabla B$ drift is away from the active $\mathrm{x}$-point. This regime reaches higher confinement than the L-mode thanks to the formation of a temperature pedestal while the density profile almost does not change. This makes it interesting to study from at least two points of view: i) transition physics, transport reduction and pedestal formation; ii) potentiality as regime in a fusion reactor.

The I-mode has been achieved in ASDEX Upgrade with NBI, ECRH and ICRF. No noticeable differences in the power threshold and confinement properties were found, except at very low density with ECRH where the decoupling between the electron and ion heat channels leads to an increase of the required ECRH power at the L-I transition as compared to NBI under similar conditions. In ASDEX Upgrade the I-mode is only achieved for the unfavourable ion 
$\nabla B$ drift because a high $\mathrm{H}$-mode threshold is required for the I-mode to develop. However, the properties of the I-mode do not depend on whether the high threshold is caused by reversing the magnetic field direction in the lower single magnetic configuration or operating in the upper single configuration. The I-mode could be obtained in a similar way independently of the wall material, carbon or tungsten, and with open or closed divertor configurations. The I-mode can be steady over several confinement times at a confinement level with $H_{98} \leq 0.8$, which requires the adequate, low enough, heating power. If the heating power is somewhat higher the normalised confinement increases beyond 0.8 , the temperature pedestal further develops spontaneously and a transition to $\mathrm{H}$-mode occurs inevitably whereby $H_{98}$ is close to unity at this transition. As the L-H power threshold increases with the magnetic field, this limitation might be less severe at higher $B_{T}$ values, as already indicated by results in C-Mod. With ECRH, $T_{e}$ is higher than $T_{i}$ in the plasma core, as compared to NBI cases, but the two temperatures are very similar at the I-mode pedestal, except with ECRH at low density where $T_{e}$ is higher than $T_{i}$ up to the edge. This is consistent with the fact that the L-I power threshold does not depend on the heating method, except at very low density with ECRH. The L-I transition seems to require a minimum ion pressure gradient at the plasma edge or a deep enough $E_{r}$ well. The L-I transition is systematically correlated with the appearance of the WCM and GAM but their possible contribution to transport reduction is not clarified yet.

Our comparison between ECRH and NBI triggered L-I transitions suggests that the ion channel plays a key role. This has a strong analogy with the results on the L-H transition in ASDEX Upgrade and then suggests that $E_{r}$ might be part of the physics mechanism, as known from the H-mode studies. However, the fact that the L-I transition depends weakly on the magnetic field value might be in contradiction with the $E \times B$ shear stabilisation hypothesis as single cause. Further investigations with a wider range of magnetic field values and accurate $E_{r}$ measurements will be required to address this important physics question.

The development of the pedestal after the L-I transition leads to a steeper edge pressure gradient and to a more negative $E_{r}$ well. This ultimately causes a transition to H-mode with the usual strong reduction of the particle transport. During the I-mode evolution the overall turbulence level at the edge decreases significantly and in ASDEX Upgrade the turbulence becomes more intermittent. Comparative studies between devices on this topic might be interesting. Overall, the I-mode provides an excellent field of investigations to better understand the physics of transport reduction and pedestal formation in comparison with the H-mode 
studies.

Thanks to its good confinement properties, without ELMs and with L-mode particle transport, the I-mode might be considered as a potential regime for future fusion reactors. However, to assess its potentiality, future studies, including multi-machine comparisons as carried out in the frame of the ITPA, should probably address at least the following topics:

1. So far the I-mode has only been obtained robustly in the unfavourable $\nabla B$ ion drift configuration to prevent the occurrence of the $\mathrm{H}$-mode and requires a higher heating power than the usual transition to H-mode. Further studies will be needed to assess whether this situation is compatible with the presently envisaged future reactors.

2. The I-mode has not yet been investigated at densities above half of the Greenwald limit. Exploring the behaviour of the I-mode towards the density limit is probably necessary. Such studies are envisaged for the forthcoming experimental campaign in ASDEX Upgrade. It is in particular proposed to transition to I-mode at low density for which the power threshold is low and then, once in the I-mode, to simultaneously increase density (with gas puffing) and heating power, remaining in the I-mode with good confinement, but avoiding the H-mode.

3. The I-mode features good confinement but rather modest $\beta_{N}$ values, while efficient fusion reactors should probably operate at higher $\beta_{N}$ than reached so far in I-modes. Further experimental studies are desirable to investigate to which extent $\beta_{N}$ can be increased in the I-mode. 4. The spontaneous uncontrolled non-steady improvement of the I-mode provides a better confinement but also leads to a transition into H-mode with large ELMs, as often observed in ASDEX Upgrade. Such a behaviour could be a serious issue for future fusion reactors and it is not clarified yet to which extent this development can be controlled or even avoided. To address this question, it will be important to understand the underlying physics mechanisms. Further, the possibilities of controlling the plasma energy and/or the pedestal development with an adequate feed-back on the heating power should also be explored. We have shown that the use of magnetic perturbations could contribute to this goal and this will be pursued. Further studies on these topics are envisaged for the next ASDEX Upgrade experimental campaign.

Overall, the I-mode features good confinement, absence of ELMs and no strong reduction of particle transport which are positive properties in view of its application to future fusion reactors but some issues should be addressed to better assess its actual potentiality for efficient and reliable operation. Due to their different operational features, the I-mode and H-mode 
possibly impose different specific technical requirements on a future fusion device.

\section{Acknowledgement}

Fruitful discussions with C. Angioni are warmly acknowledged. This work has been partly carried out within the framework of the EUROfusion Consortium and has received funding from the Euratom research and training programme 2014-2018 under grant agreement number 633053. The views and opinions expressed herein do not necessarily reflect those of the European Commission.

[1] F. Ryter et al., in Europhysics Conference Abstracts (Proc. of the 22th EPS Conference on Controlled Fusion and Plasma Physics, Bournemouth, 1995), edited by B. Keen et al., volume 19C, part IV, pages 89-92, Geneva, 1995, EPS.

[2] ITER Physics Basis, Chap. II, page 2190, Nucl. Fusion 39 (1999).

[3] P. N. Yushmanov et al., Nucl. Fusion 30 (1990) 1999.

[4] H-MODE DATABASE WORKING GROUP presented by KARDAUN O.J.W.F., Plasma Physics and controlled nuclear fusion research, proceedings of the 14th IAEA Conf. Plasma Phys Contr Fus. (1993).

[5] F. Ryter et al., Plasma Physics and Controlled Fusion 40 (1998) 725.

[6] R. M. McDermott et al., Physics of Plasmas 16 (2009) 056103.

[7] D. Whyte et al., Nuclear Fusion 50 (2010) 105005.

[8] A. E. Hubbard et al., Physics of Plasmas 18 (2011) 056115.

[9] A. Hubbard et al., Nuclear Fusion 52 (2012) 114009.

[10] G. D. Conway et al., Phys. Rev. Lett. 106 (2011) 065001.

[11] G. Birkenmeier et al., Nuclear Fusion 56 (2016) 086009.

[12] A. White et al., Nuclear Fusion 51 (2011) 113005.

[13] I. Cziegler et al., Physics of Plasmas (1994-present) 20 (2013) .

[14] P. Manz et al., Nuclear Fusion 55 (2015) 083004.

[15] F. Leuterer et al., Nuclear Fusion 43 (2003) 744.

[16] A. Hubbard et al., Nuclear Fusion 56 (2016) 086003.

[17] A. Mlynek et al., Review of Scientific Instruments 81 (2010) 033507.

[18] M. Willensdorfer et al., Plasma Physics and Controlled Fusion 56 (2014) 025008.

[19] R. Fischer et al., Fusion Science and Technology 58 (2010) 675.

[20] S. K. Rathgeber et al., Plasma Physics and Controlled Fusion 55 (2013) 025004.

[21] R. M. McDermott et al., Plasma Physics and Controlled Fusion 53 (2011) 124013.

[22] E. Viezzer et al., Review of Scientific Instruments 83 (2012) 103501.

[23] K. H. Burrell et al., Plasma Phys. Controlled Fusion 31 (1989) 1649.

[24] R. J. Groebner et al., Phys. Rev. Lett. 64 (1990) 3015.

[25] E. Viezzer et al., Nuclear Fusion 53 (2013) 053005.

[26] A. Silva et al., Review of Scientific Instruments 70 (1999) 1072. 
[27] L. Cupido et al., Review of Scientific Instruments 75 (2004) 3865.

[28] G. D. Conway et al., Plasma Physics and Controlled Fusion 46 (2004) 951.

[29] G. D. Conway, Plasma Physics and Controlled Fusion 50 (2008) 124026.

[30] ITER Physics Basis, Chap. II, page 2208, Table 5, Nucl. Fusion 39 (1999).

[31] M. Bernert et al., Review of Scientific Instruments 85 (2014) 033503.

[32] H. Biglari et al., Phys. Fluids B 2 (1990) 1.

[33] F. L. Hinton et al., Rev. Mod. Phys. 48 (1976) 239.

[34] F. Wagner, Plasma Physics and Controlled Fusion 49 (2007) B1.

[35] P. Sauter et al., Nuclear Fusion 52 (2012) 012001.

[36] C. Theiler et al., Nuclear Fusion 54 (2014) 083017.

[37] T. Happel et al., Nuclear Fusion 56 (2016) 064004.

[38] T. Happel et al., in 43rd EPS Conference on Plasma physics, paper I1.101, 2016, submitted to Plasma Phys. Control. Fus.

[39] W. Suttrop et al., Phys. Rev. Lett. 106 (2011) 225004.

[40] W. Suttrop et al., Plasma Physics and Controlled Fusion 53 (2011) 124014.

[41] F. Ryter et al., in Europhysics Conference Abstracts (CD-ROM, Proc. of the 38th EPS Conference on Plasma Physics, Strasbourg, France, 2011), edited by A. Becoulet et al., volume 35G of ECA, page P5.112, Geneva, 2011, European Physical Society.

[42] Y. R. Martin et al., Journal of Physics: Conference Series 123 (2008) 012033.

[43] F. Ryter et al., Nuclear Fusion 52 (2012) 114014.

[44] F. Ryter et al., Nuclear Fusion 53 (2013) 113003.

[45] F. Ryter et al., Nuclear Fusion 54 (2014) 083003.

[46] F. Ryter et al., Nuclear Fusion 36 (1996) 1217.

[47] M. Cavedon, in Europhysics Conference Abstracts (CD-ROM, Proc. of the 42th EPS Conference on Plasma Physics, Lisbon, Portugal, 2015), edited by R. Bingham et al., volume 39E of ECA, page P1.130, Geneva, 2015, European Physical Society.

[48] U. Stroth et al., Plasma Physics and Controlled Fusion 53 (2011) 024006.

[49] F. Ryter et al., Plasma Physics and Controlled Fusion 58 (2016) 014007.

[50] A. Marinoni et al., Nuclear Fusion 55 (2015) 093019.

[51] P. Gohil et al., Nuclear Fusion 50 (2010) 064011.

[52] S. Kaye et al., Nuclear Fusion 37 (1997) 1303.

[53] J.R. Walk, Private communication 2015.

[54] W. Suttrop et al., Plasma Physics and Controlled Fusion 39 (1997) 2051.

[55] F. Ryter et al., Nuclear Fusion 41 (2001) 537.

[56] G. Tardini et al., Nuclear Fusion 42 (2002) 258. 